\title{
Human prostate luminal cell differentiation requires NOTCH3 induction by p38-MAPK and MYC
}

\author{
Sander B. Frank ${ }^{1,2,3}$, Penny L. Berger ${ }^{1}$, Mats Ljungman ${ }^{4}$ and Cindy K. Miranti ${ }^{1,3, *}$
}

\begin{abstract}
Many pathways dysregulated in prostate cancer are also involved in epithelial differentiation. To better understand prostate tumor initiation, we sought to investigate specific genes and mechanisms required for normal basal to luminal cell differentiation. Utilizing human prostate basal epithelial cells and an in vitro differentiation model, we tested the hypothesis that regulation of NOTCH3 by the p38 MAPK family (hereafter p38-MAPK), via MYC, is required for luminal differentiation. Inhibition (SB202190 and BIRB796) or knockdown of p38 $\alpha$ (also known as MAPK14) and/or p38 (also known as MAPK13) prevented proper differentiation. Additionally, treatment with a $\gamma$ secretase inhibitor (RO4929097) or knockdown of $\mathrm{NOTCH} 1$ and/or $\mathrm{NOTCH} 3$ greatly impaired differentiation and caused luminal cell death. Constitutive p38-MAPK activation through MKK6(CA) increased NOTCH3 (but not NOTCH1) mRNA and protein levels, which was diminished upon MYC inhibition (10058-F4 and JQ1) or knockdown. Furthermore, we validated two NOTCH3 enhancer elements through a combination of enhancer (e)RNA detection (BruUV-seq) and luciferase reporter assays. Finally, we found that the NOTCH3 mRNA half-life increased during differentiation or upon acute p38-MAPK activation. These results reveal a new connection between p38-MAPK, MYC and $\mathrm{NOTCH}$ signaling, demonstrate two mechanisms of $\mathrm{NOTCH} 3$ regulation and provide evidence for $\mathrm{NOTCH} 3$ involvement in prostate luminal cell differentiation.
\end{abstract}

KEY WORDS: Prostate, Luminal cell differentiation, NOTCH, MYC, p38-MAPK, Development

\section{INTRODUCTION}

The human prostate gland contains an epithelial bilayer of basal and luminal cells. Within these layers resides a combination of uni- and bi-potent progenitors important for normal gland homeostasis (Kwon et al., 2016; Ousset et al., 2012; Uzgare et al., 2004). Basal and luminal cells display distinct markers, such as androgen receptor (AR) and keratin 8 (K8; also known as KRT8) in the luminal layer, and laminin-binding integrins and keratin 5 (K5; also known as KRT5) in the basal layer (Lamb et al., 2010). Human prostate tumors co-express some of the basal and luminal markers, suggesting a defect in differentiation (Tokar et al., 2005). Moreover, many of the commonly altered genes in prostate cancer (e.g. $M Y C$,

\footnotetext{
${ }^{1}$ Laboratory of Integrin Signaling and Tumorigenesis, Van Andel Research Institute Grand Rapids, MI 49503, USA. ${ }^{2}$ Genetics Program, Michigan State University, East Lansing, MI 48824, USA. ${ }^{3}$ Department of Cellular and Molecular Medicine, University of Arizona Cancer Center, Tucson, AZ 85724, USA. ${ }^{4}$ Translational Oncology Program, University of Michigan Medical School, Ann Arbor, MI 48109, USA.

*Author for correspondence (cmiranti@email.arizona.edu)

(D) C.K.M., 0000-0003-0668-6106
}

Received 2 September 2016; Accepted 17 April 2017
$A R, E R G$ and PTEN) are also implicated in differentiation (Frank and Miranti, 2013). We previously demonstrated that manipulation of differentiation regulators (MYC, PTEN and ING4) in normal human prostate epithelial cells results in tumor formation when grafted into a mouse prostate (Berger et al., 2014). To better understand tumor initiation in prostate epithelium, we sought to investigate specific genes and mechanisms required for normal basal to luminal cell differentiation.

The p38 MAPK family (hereafter p38-MAPK) is a known driver of epithelial differentiation in various tissues including skin and lung (Cuadrado and Nebreda, 2010). p38-MAPK regulates a wide range of targets, including other kinases/phosphatases, transcription factors and RNA-binding proteins (Cuadrado and Nebreda, 2010). Moreover, p38-MAPK is a downstream target of FGFR2b, a crucial receptor for epithelial differentiation in the skin and prostate (Belleudi et al., 2011; Heer et al., 2006; Lamb et al., 2010). Despite these findings, how p38MAPK expression in prostate epithelial cells drives differentiation, including its relevant targets, remains poorly defined.

MYC positively regulates normal skin and prostate differentiation, and is a major prostate cancer oncogene (Berger et al., 2014; Gebhardt et al., 2006; Koh et al., 2010). MYC potentially targets thousands of genes via its activity as a transcription factor, and many of its targets are tissue and context specific (Conacci-Sorrell et al., 2014; Lüscher and Vervoorts, 2012). In normal prostate, transient upregulation of MYC is required for loss of cell adhesion and stimulation of chromatin remodeling (Berger et al., 2014). Moreover, regulation of MYC itself is complex, occurring at many different levels including pre- and post-transcription and through post-translational modification (McKeown and Bradner, 2014). Overexpression of AR in human primary basal prostate epithelial cells is sufficient to cause growth arrest via transcriptional downregulation of MYC (Antony et al., 2014; Vander Griend et al., 2014). Thus, MYC plays a crucial role in multiple aspects of both normal prostate differentiation and cancer.

NOTCH controls cell fate, including stemness, survival and differentiation (Deng et al., 2015). Mammals express four NOTCH transmembrane receptors (NOTCH1- NOTCH4), five canonical transmembrane ligands (JAG1 and JAG2, and DLL1, DLL3 and DLL4) and ten classic downstream targets (HES1-HES7, HEY1, HEY2 and HEYL). Cell-cell contact joins ligand and receptor, triggering proteolytic cleavage of NOTCH by the $\gamma$-secretase complex which releases the active intracellular domain (ICD) of the receptor into the nucleus to activate transcription (Kopan and Ilagan, 2009). NOTCH can promote cell cycle arrest and de-adhesion from the matrix, both of which are essential for luminal differentiation (Hodkinson et al., 2007; Mazzone et al., 2010; Rangarajan et al., 2001). Furthermore, NOTCH1 signaling can promote survival of human basal cells (Dalrymple et al., 2005; Litvinov et al., 2006). In a mouse model, constitutively active NOTCH1 driven by a luminal promoter causes prostatic intraepithelial neoplasia (PIN) and increases survival of a subset of luminal cells in 3D culture (Kwon et al., 2014; Valdez et al., 2012). However, there are conflicting reports as to 
whether the NOTCH pathway is oncogenic or tumor suppressive, and the specific role for the other NOTCH receptors remains undefined (Carvalho et al., 2014; Kwon et al., 2016).

We sought to understand how p38-MAPK, MYC and NOTCH work together in normal prostate differentiation. We utilized an established model of in vitro differentiation of human basal prostate epithelial cells (PrECs) (Berger et al., 2014, 2017; Lamb et al., 2010). By using pharmacologic and genetic manipulation, we tested the hypothesis that p38-MAPK upregulation of $\mathrm{NOTCH3}$, via $\mathrm{MYC}$, is required for efficient induction and maintenance of the suprabasal layer during prostate differentiation. We identify two mechanisms of NOTCH3 regulation by p38-MAPK, both at the transcriptional and post-transcriptional level. This knowledge improves our understanding of prostate epithelial differentiation by tying together multiple pathways and elucidating new mechanisms for key differentiation regulators.

\section{RESULTS}

p38-MAPK isoforms $p 38 \alpha$ and $p 38 \delta$ are required for prostate epithelial differentiation

PrECs were induced to differentiate by treating with keratinocyte growth factor (KGF; also known as FGF7) and synthetic androgen
(R1881) for 2 weeks (Lamb et al., 2010). This results in a stratified epithelium consisting of suprabasal luminal cells sitting on top of basal cells. p38-MAPK is a known downstream target of KGF-toFGFR2 signaling and is implicated in epithelial differentiation in several tissue types, including prostate (Belleudi et al., 2011; Lamb et al., 2010). Four different genes encode p38-MAPK isoforms:

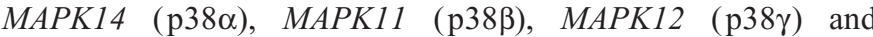

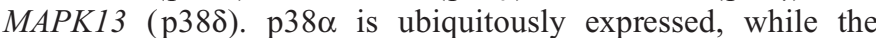
other isoforms are typically more tissue specific (Cuadrado and Nebreda, 2010). RNA-seq and immunoblotting identified p38 $\alpha$ and $\mathrm{p} 38 \delta$ to be the predominantly expressed isoforms in basal PrECs (Fig. 1A,B).

Lysates from differentiating cells were collected over a 2-week time course, and p38 $\alpha$ activity was measured by immunoblotting with an antibody specific for its activated phosphorylated form ( $p-p 38 \alpha)$. In primary cells (PrECs), elevated p-p38 $\alpha$ was detected at day 4 and remained elevated (Fig. 1C). In immortalized cells (iPrECs), which take 4 days longer to differentiate, p-p38 $\alpha$ was elevated at day 8 (Fig. 1D). Semi-quantification of a set of biological triplicate experiments indicates that both total p38o and p-p38 $\alpha$ levels increase $\sim 2$-fold at day 4 and $\sim 3$-fold by day 12 (Fig. S1A,B).
A

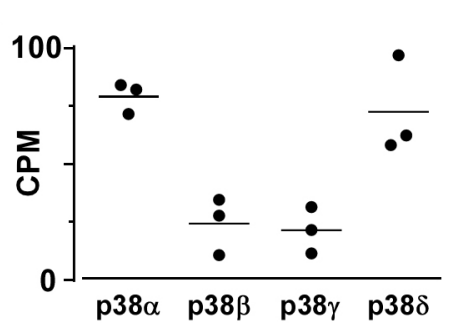

B
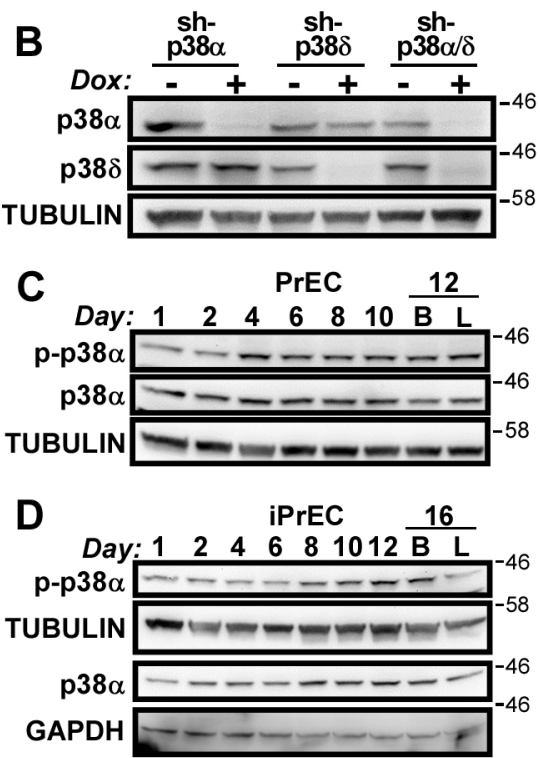
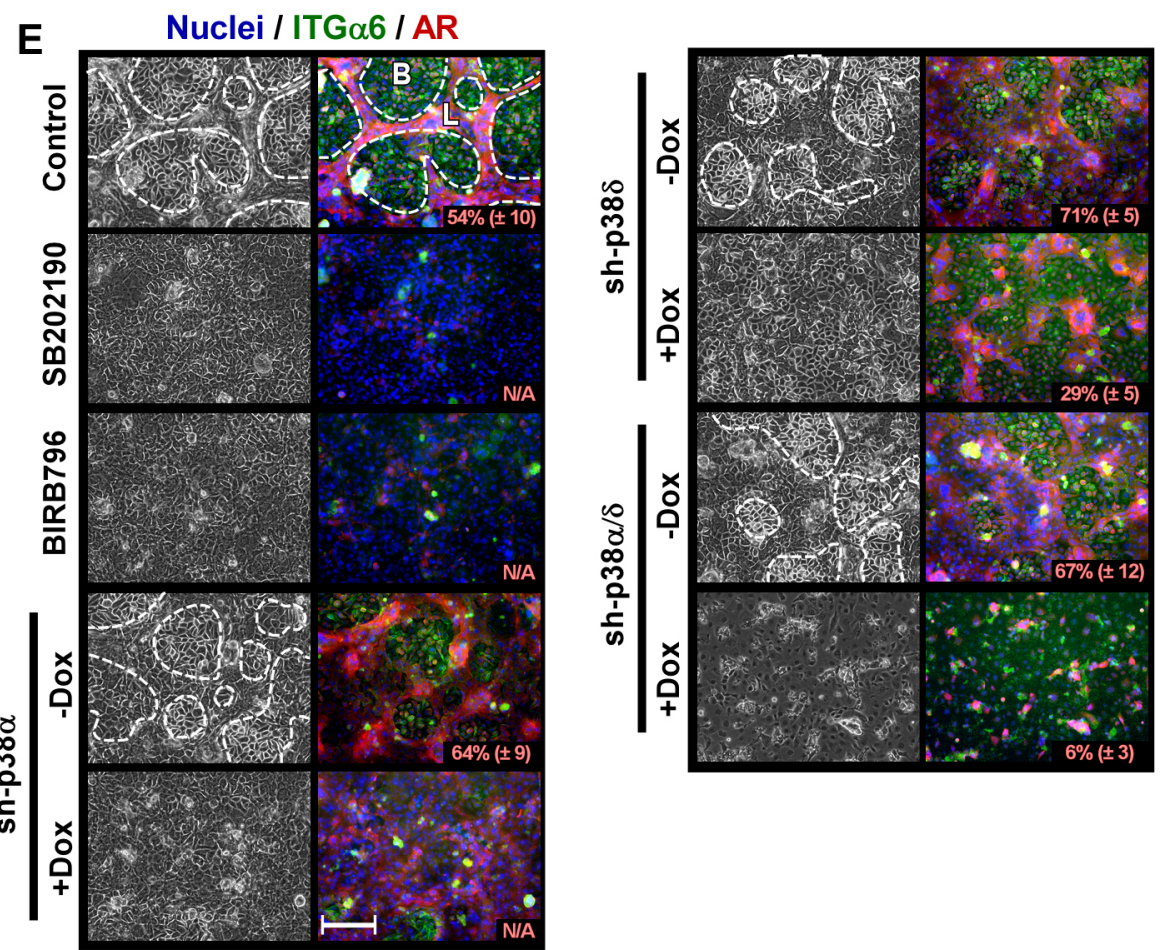

Fig. 1. p38 $\alpha$ - and p38 data of basal iPrECs. Line indicates mean of biological triplicates. (B) Lysates from stable pools of iPrECs expressing Tet-inducible p38 $\alpha$ and/or p38 shRNAs differentiated for 16 days with or without Dox and probed by immunoblotting. Numbers on right indicate the position of molecular mass markers ( $\mathrm{kDa}$ ). (C,D) Primary (PrECs) and immortalized (iPrECs) cells differentiated with KGF and R1881, and lysates collected at indicated time points for immunoblotting. Luminal cells $(\mathrm{L})$ were separated from the basal cells $(B)$ at the final time point before lysis. Note that the same lysates were run on multiple gels for Fig. 1D and Fig. 2A. (E) iPrECs were differentiated for 16 days with DMSO and Dox (control), $1 \mu \mathrm{M}$ SB202190 or $0.1 \mu \mathrm{M}$ BIRB796, while inducible shRNA lines were treated with or without Dox. Left columns, phase-contrast images. Right columns: merged epifluorescence images (10× objective) of Hoescht-33258-stained nuclei (blue), immunostaining for androgen receptor ( $A R$, red) and integrin $\alpha 6$ (ITG $\alpha 6$, green). AR is luminal (L) and ITG $\alpha 6$ is basal (B). The upper layer is outlined (dashed line). The percentage in the lower right corner is the mean \pm s.d. area of coverage by suprabasal cells from three fields. N/A, no clearly defined suprabasal layer. Scale bar: $200 \mu \mathrm{m}$ 
To determine whether p38-MAPK is necessary for differentiation, iPrECs were differentiated in the presence of two p38-MAPK inhibitors (SB202190 and BIRB796) or Dox-induced shRNA against p38 $\alpha$ (sh-p38 $\alpha$ ), p38 (sh-p38 ) or both (sh-p38 $\alpha / \delta)$. Inhibitor concentrations were selected based on their ability to block CREB1 phosphorylation mediated by constitutively active MKK6 [MKK6(CA); MKK6 is also known as MAP2K6] (Fig. S1C). Effective knockdown of $\mathrm{p} 38 \alpha$ and/or p38 by shRNA was verified by immunoblotting (Fig. 1B). After 16 days of differentiation, control cells (Dox plus DMSO) differentiated normally, as measured by loss of integrin $\alpha 6$ and gain in AR, with a $54 \%$ coverage of the culture by suprabasal cells (averaged from three fields) (Fig. 1E). Treatment with $1 \mu \mathrm{M}$ SB202190 or $0.1 \mu \mathrm{M}$ BIRB796 completely prevented formation of an AR-positive suprabasal layer. Unexpectedly, integrin $\alpha 6$ (ITG $\alpha 6$ ) expression was also decreased by these inhibitors. However, this was not due to basal cell toxicity (as judged by the lack of cleaved caspase 3) nor decreased proliferation (as demonstrated by measuring BrdU incorporation) (Fig. S1D,E). Dox-induced shRNA knockdown of $\mathrm{p} 38 \alpha$ did not prevent AR-positive cells from appearing, but it did prevent formation of a distinct suprabasal layer. On the other hand, knockdown of $\mathrm{p} 38 \delta$ reduced the production of cells that were both $\mathrm{AR}^{+}$and ITGo6 $6^{-}(29 \%$ suprabasal coverage, reduced from $71 \%$ ), but did not completely block it. However, double p38 $\alpha / \delta$ knockdown drastically prevented suprabasal layer formation $(6 \%$ suprabasal coverage,

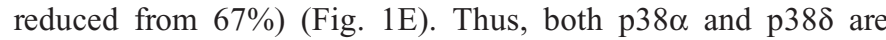
required for normal luminal cell differentiation, and the differing effects of their loss suggests they may control different steps in suprabasal layer formation.

\section{NOTCH3 is induced during differentiation}

A hallmark of normal luminal cell differentiation is the downregulation of integrins including $\alpha 6, \alpha 3, \quad \beta 4$ and $\beta 1$. $\mathrm{NOTCH}$ can negatively regulate integrin expression and is generally required for epithelial differentiation (Frank and Miranti, 2013; Koh et al., 2010; Mazzone et al., 2010). Additionally, MYC suppresses integrin $\alpha 6$ and $\beta 1$ expression (Gebhardt et al., 2006), and was previously demonstrated to be required for prostate differentiation (Berger et al., 2014). In some contexts, MYC is a direct downstream target of NOTCH (Weng et al., 2006). To decipher the roles of MYC and NOTCH, lysates from differentiating iPrECs (Fig. 2A) or primary PrECs (Fig. S2A) were collected over a 2-week time course and protein expression measured by immunoblotting. MYC expression and activation (phosphorylation; denoted p-MYC) was initially elevated but waned as basal cell proliferation subsided and transiently elevated again at around day 8 (Fig. 2A). A similar response was observed in primary cells but it occurred 4 days earlier, as expected due to their faster differentiation (Fig. S2A).

Of the four NOTCH receptors, we were only able to detect significant expression of NOTCH1, NOTCH2 and NOTCH3 (Fig. 2A). Expression of NOTCH2 remained essentially unchanged during differentiation. NOTCH1 protein was initially high, then decreased slightly. In contrast, NOTCH3 protein expression was very low in basal cells, then increased with time during differentiation; moreover, a marked increase occurred at around day 8, when $\mathrm{p} 38 \alpha$ and MYC activity were also maximal (Fig. 2A). A similar pattern was observed in primary PrECs at day 4 (Fig. S2A).

NOTCH1 and NOTCH3 mRNA expression, as measured by quantitative real-time PCR (qRT-PCR), paralleled protein expression; NOTCH1 dipped and recovered to baseline levels, while NOTCH3 increased dramatically and remained higher in the suprabasal layer (Fig. 2B). NOTCH3 mRNA appeared to increase in two phases; a steady climb increasing $\sim 10$-fold over the first 8 days followed by a more dramatic spike, up $\sim 220$-fold by day 14 in the suprabasal cells (Fig. 2B). NOTCH ligands also displayed two distinct expression profiles; $J A G 1$ (Fig. 2B) and DLL4 (Fig. S2B) showed initial decreases but then recovered by day 10 , following the pattern of NOTCH1 expression. Meanwhile, DLL3 remained flat and began to increase after day 10, paralleling the increase in NOTCH3 mRNA expression (Fig. 2B). HEY2, HEYL (Fig. 2B), HES1, HES6 and HEY1 (Fig. S2B) all increased during differentiation, with day 8 being a key inflection point. HEY2 mRNA was unique in that it segregated into the suprabasal population (up 45-fold versus day 1) similar to NOTCH3. These data indicate that the day 8-10 window is critical for activation of the NOTCH pathway, and correlates with the appearance of an emerging suprabasal layer and integrin $\alpha 6 \beta 1$ mRNA downregulation (Fig. S2B).

\section{NOTCH1 and NOTCH3 are required for differentiation}

To examine the requirement of NOTCH1 and/or NOTCH3 for differentiation, iPrECs were differentiated and treated with either a $\gamma$-secretase inhibitor (RO4929097) or Dox to induce expression of NOTCH1 and/or NOTCH3 shRNA. Efficient knockdown of NOTCH1 and/or NOTCH3 mRNA was achieved by $48 \mathrm{~h}$ (Fig. S2C) and protein at $96 \mathrm{~h}$ (Fig. S2D). NOTCH3 loss also led to a slight decrease in NOTCH1 protein; however, this was not due to an off-target shRNA effect on NOTCH1 since NOTCH1 mRNA was not affected (Fig. S2C,D). Control and non-Dox-treated cells differentiated normally as indicated by formation of a suprabasal layer of cells (both $\mathrm{AR}^{+}$and $\mathrm{ITG} 6^{-} ; 44-53 \%$ coverage), while treatment with RO4929097 ablated differentiation (Fig. 2C). Induced knockdown of NOTCH1 or NOTCH3 by means of shRNA each led to disruption of the suprabasal layer, with $16 \%$ and $31 \%$ coverage respectively, compared to $53 \%$ and $44 \%$ for control cells. Double knockdown of NOTCH1 and NOTCH3 more severely disrupted differentiation, giving a similar appearance to that seen upon treatment with RO4929097 (Fig. 2D). Furthermore, propidium iodide staining indicated that the suprabasal cell clumps observed upon NOTCH inhibition or knockdown were mostly dead cells (Fig. S2E). Thus, NOTCH1 and NOTCH3 are both required for survival of the suprabasal cells during luminal cell differentiation.

\section{p-p38 and NOTCH3 are expressed and active in early differentiating cells}

iPrECs were immunostained for p-p38 (all p38-MAPK isoforms) and $\mathrm{NOTCH} 3$ at key times during differentiation to observe expression levels and localization (Fig. 2E). Nuclear p-p38 was detected in all basal cells at day 4, when very little NOTCH3 expression was detected, except for in a few cells where it was nuclear localized. By day 8 , patches of more intense p-p38 nuclear staining were detected, which corresponded to cells in which NOTCH3 levels were dramatically increased (white arrow). NOTCH3 localization was primarily nuclear in the basal cells, but both nuclear and cytoplasmic staining was apparent at days 8 and 12 , when suprabasal layer formation is maximal. By day 21, more membrane and less nuclear staining was observed, with staining occurring primarily in the suprabasal cells with very low levels in the basal cells. p-p38 nuclear localization was lost as suprabasal cells became established. Thus, p-p38 nuclear activity peaks around day 8 , just as NOTCH3 expression and downstream signaling 

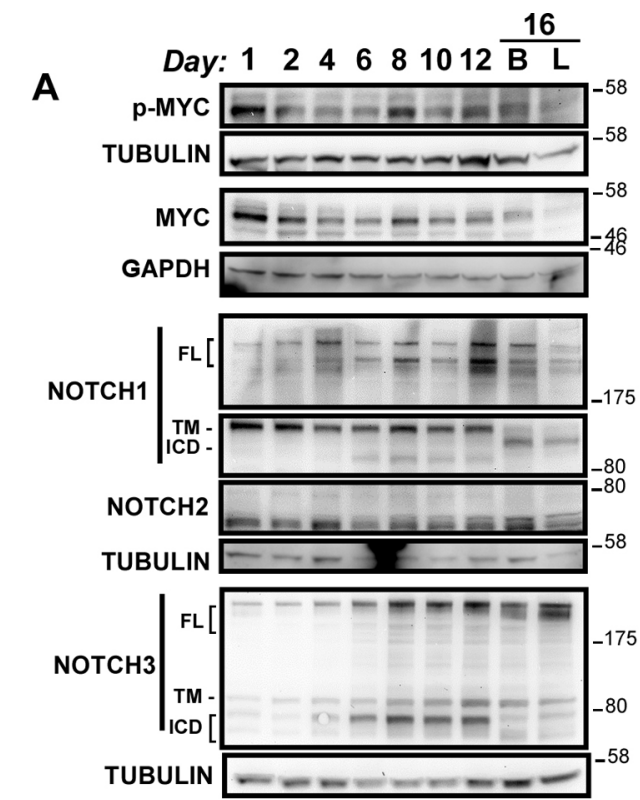

B



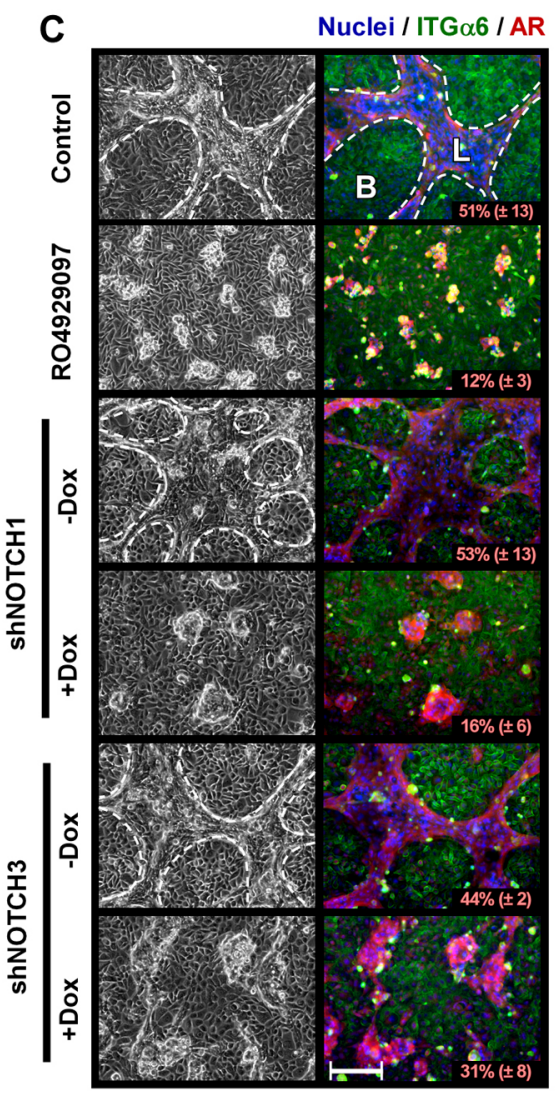



D Nuclei $/$ ITG $\alpha 6 /$ AR

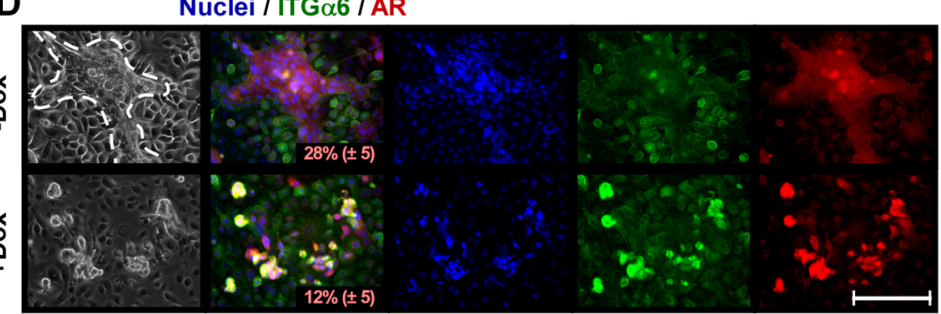

$\mathbf{E}$

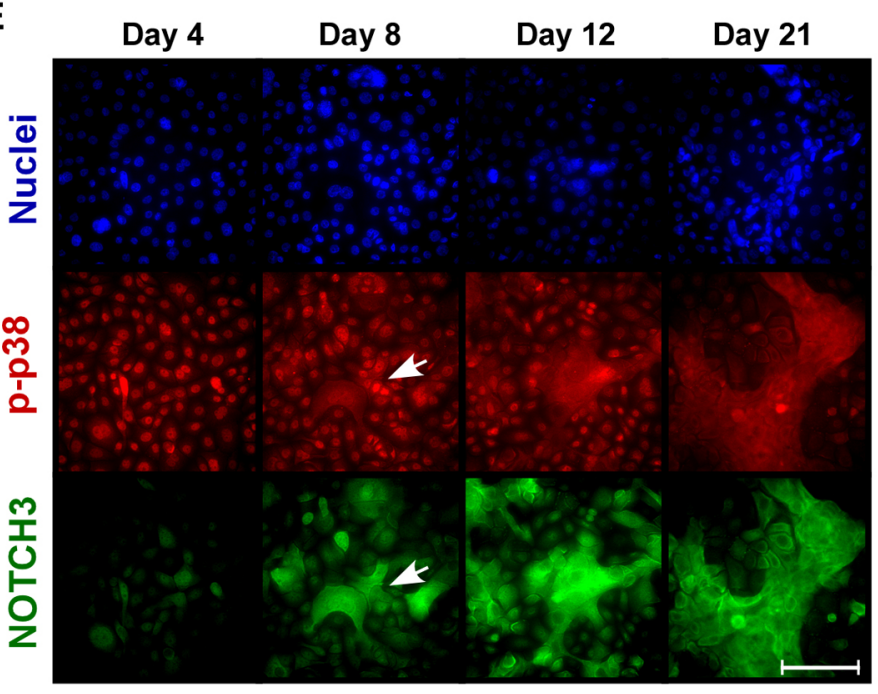

Fig. 2. NOTCH1 and NOTCH3 are required for differentiation. (A) iPrECs were differentiated and analyzed by immunoblotting as in Fig. 1D. Note that the antibody used in the P-MYC row recognizes MYC phosphorylation at T58 and S62, and the antibody used in the NOTCH2 row is specific to the ICD. The antibody used for NOTCH1 and NOTCH3 recognize full-length (FL), transmembrane (TM) and intracellular domain (ICD) forms. Also note that the same lysates were run on multiple gels for Fig. 1D and Fig. 2A. (B) RNA was collected for qRT-PCR to analyze ligands and downstream targets of NOTCH during differentiation. Luminal ( $\mathrm{L}$, solid line) cells were separated from basal (B, dashed line) cells at days 10 and 14. Data were normalized to values at day 1. Graph shows mean \pm s.d. of biological triplicates. (C) iPrECs were differentiated for 12 days with DMSO and Dox (control) or $1 \mu$ M RO4929097, while shRNA lines were treated with or without Dox. The percentage in the lower right corner is mean \pm s.d. area of coverage by suprabasal cells from three fields. Left column, phase-contrast iamges. Right column, merged epifluorescence images (10x objective) of Hoescht-33258-stained nuclei (blue), immunostaining for AR (red) and ITG 6 (green). The upper layer is outlined (dashed line). The percentage in the lower right corner is mean \pm s.d. area of coverage by suprabasal cells from three fields. (D) iPrECs with shRNAs against NOTCH1 and NOTCH3 were differentiated with KGF and $1 \mathrm{nM}$ R1881 for 21 days and imaged with a $20 \times$ objective. (E) iPrECs were differentiated for various times and immunostained for p-p38 (red) and NOTCH3 (green). Note that the anti-p-p38 antibody is not specific for an isoform. The arrows indicate cell clusters co-expressing elevated p-p38 and NOTCH3. Scale bars: $200 \mu \mathrm{m}$. 
increases in the suprabasal layers. Once established, NOTCH3 expression remains high in the suprabasal layer and p-p38 is lost from the nucleus.

\section{MKK6-induced p38-MAPK activation recapitulates differentiation-induced MYC and NOTCH3 expression}

To determine the relationship between p38-MAPK and NOTCH3, we engineered an iPrEC line with a Dox-inducible constitutively active MKK6 mutant, MKK6(CA), which directly phosphorylates and activates p38-MAPK (Alonso et al., 2000). During differentiation, p38-MAPK activation is moderately elevated over several days (Fig. S1A,B), but when MKK6(CA) is induced, the signaling events that naturally occur over days are condensed into hours (Fig. 3A). Although prolonged constitutive p38-MAPK activation leads to stress and cell death, the Dox-inducible system allows us to tightly control induction and measure downstream signaling over a short time period. A $16 \mathrm{~h}$ treatment of iPrECTetON-MKK6(CA) cells with Dox led to an $~ 18$-fold increase in NOTCH3 mRNA (Fig. 3B). Conversely, MKK6(CA) induction decreased NOTCH1 by $\sim 2.5$-fold. Inhibition of p38-MAPK blocked these effects (Fig. 3B).

To establish a temporal order of events, iPrEC-TetON-MKK6 (CA) cells were treated with Dox and lysates collected over time (Fig. 3C). MKK6(CA) was detectable as early as $4 \mathrm{~h}$, at which time a corresponding increase in active p-p38 $\alpha$ and MYC was observed, peaking at $\sim 7-8 \mathrm{~h}$. NOTCH3 levels began to increase around $6 \mathrm{~h}$ and continued to climb. At the mRNA level, $M Y C$ induction also preceded an increase in NOTCH3 and decrease in NOTCH1 (Fig. 3D). Furthermore, a short pulse of Dox was sufficient to induce NOTCH3 to higher levels than normally seen at day 4 of differentiation (Fig. 3E); meanwhile, expression of NOTCH1 was decreased. These results show that constitutive activation of MKK6 is sufficient to induce $\mathrm{p} 38 \alpha$, MYC, MYC phosphorylation and NOTCH3, while downregulating NOTCH1. Thus, the MKK6(CA) model mimics the regulation of these genes observed in the standard differentiation assay. Moreover, differentiation of iPrECs for 4 days in the presence of a p38-MAPK inhibitor suppressed MYC induction and dampened NOTCH3 upregulation ( 7-vs $\sim 28$-fold),
A



C

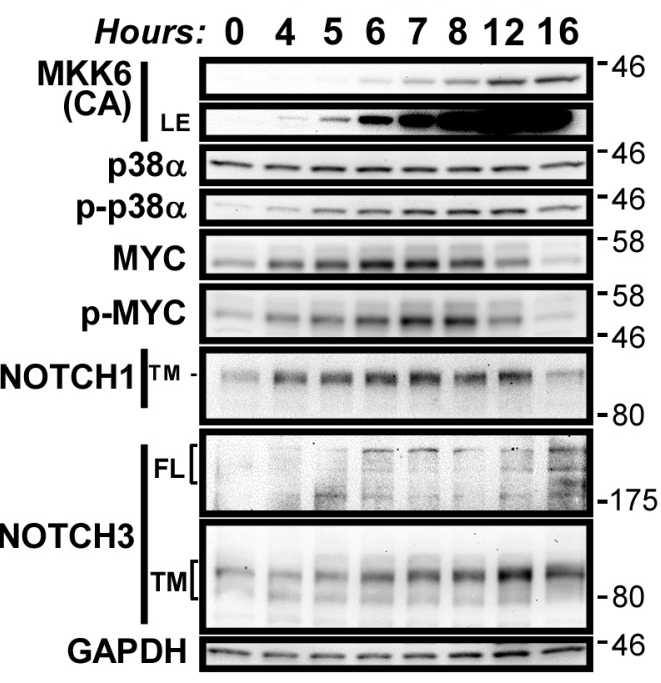

Normal Differentiation

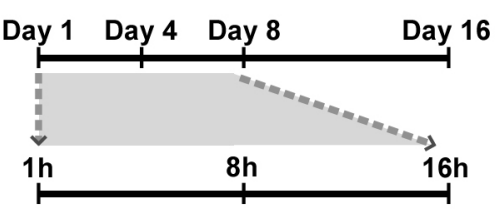

B



D

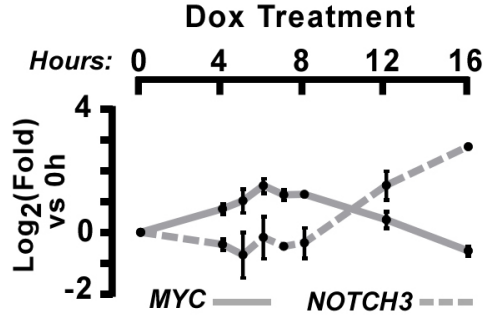

E

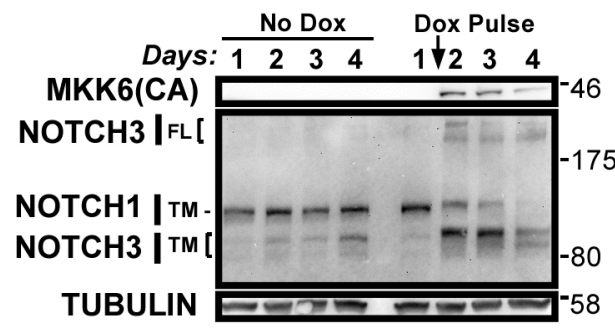

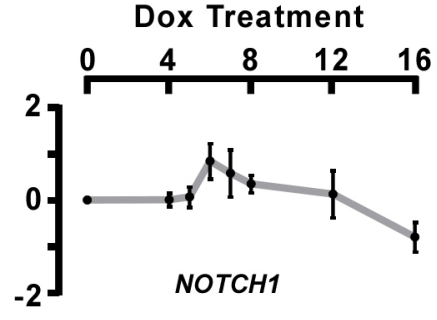

$\mathbf{F}$



Fig. 3. p38-MAPK induces NOTCH3. (A) Diagram of the Tet-inducible MKK6(CA) model. iPrECs were engineered to stably express a Dox-inducible constitutively active MKK6 mutant, MKK6(CA), which phosphorylates and activates all p38 isoforms. Acute p38-MAPK activation condenses early differentiation signaling events from days into hours. (B) iPrEC-TetON-MKK6(CA) cells were treated with or without Dox for 16 h with DMSO or $5 \mu M$ SB202190, and analyzed by qRT-PCR. Data were normalized to cells without Dox (DMSO-only). Graph shows mean \pm s.d. of biological triplicates. Numbers in bars indicate fold change. (C) iPrEC-TetOn-MKK6(CA) cells were treated with Dox for up to $16 \mathrm{~h}$ and harvested at indicated times for immunoblotting. LE, long exposure; FL, full length; TM, transmembrane. (D) iPrEC-TetON-MKK6(CA) cells were treated as in C and analyzed by qRT-PCR. Data were normalized to $0 \mathrm{~h}$ samples. Graph shows mean \pm s.d. of biological triplicates. (E) iPrEC-TetON-MKK6(CA) cells were differentiated for 1-4 days with or without a $4 \mathrm{~h}$ pulse of Dox after day 1 (arrow), and analyzed by immunoblotting. Note: NOTCH1 was probed after NOTCH3 and both are shown on the same blot. (F) iPrECs were differentiated for 4 days with DMSO or $5 \mu$ M SB202190, and analyzed by qRT-PCR. Data were normalized to the value at day 1. Graph shows mean \pm s.d. of biological triplicates. Numbers in bars indicate fold change. ${ }^{* *} P \leq 0.01,{ }^{* *} P \leq 0.001$. 
thus confirming their roles downstream of p38-MAPK in this model (Fig. 3F).

\section{MYC is required for p38-MAPK regulation of NOTCH3}

Induction of NOTCH3 mRNA by p38-MAPK could be due to direct activation of an existing transcription factor or indirect, requiring synthesis of a new factor. iPrEC-TetON-MKK6(CA) cells were treated with Dox for $12 \mathrm{~h}$ and cyclohexamide (CHX) was added at 6,8 or $10 \mathrm{~h}$ to measure the requirement for new protein synthesis. Addition of $\mathrm{CHX}$ at $6 \mathrm{~h}$ blocked NOTCH3 mRNA upregulation, while addition at $8 \mathrm{~h}$ or later did not (Fig. 4A; Fig. S3A). Thus, there is a requirement for the synthesis of an intermediate, which must be translated between 6 and $8 \mathrm{~h}$ after Dox; this matches the time of maximal MYC induction and activation (see Fig. 3C).

To test whether NOTCH3 induction requires MYC, iPrECTetON-MKK6(CA) cells were transfected with siRNA against MYC (denoted si.MYC) or a non-targeting control sequence and induced with Dox for $12 \mathrm{~h}$. MYC mRNA was knocked down $\sim 80 \%$ and NOTCH3 mRNA induction was half that seen in the control cells (5- vs 10-fold) (Fig. 4B). Similar results were observed at the protein level as assessed by immunoblotting (Fig. 4C). To further address the dependency of NOTCH3 induction on MYC, we utilized an antagonist of the MYC-MAX complex, 10058-F4 (Huang et al., 2006). iPrEC-TetON-MKK6(CA) cells were treated with Dox and increasing concentrations of $10058-\mathrm{F} 4$ for $16 \mathrm{~h}$. Treatment with as little as $5 \mu \mathrm{M} 10058-\mathrm{F} 4$ suppressed the induction of NOTCH3 protein (Fig. 4D), whereas $20 \mu \mathrm{M}$ was required to suppress NOTCH3 mRNA (Fig. S3B). These doses are at or below common usage for 10058-F4 (Guo et al., 2009; Wang et al., 2014). As an alternative approach, we used JQ-1, a BET bromodomain inhibitor, to block transcription of MYC (Delmore et al., 2011). JQ-1 prevented MKK6(CA)-induced MYC and NOTCH3 expression at 100-500 nM (Fig. 4E). Taken together, these results demonstrate that MYC is required for maximal p38MAPK-mediated induction of NOTCH3.

To determine whether MYC is sufficient for NOTCH3 induction, we generated a Tet-inducible MYC-expressing cell line: iPrECTetON-MYC. MYC induction occurred within $2 \mathrm{~h}$ of Dox treatment and NOTCH3 protein increased slightly by $6 \mathrm{~h}$ (Fig. 4F). However, there was no change in NOTCH3 mRNA (Fig. S3C). We also induced MYC after first differentiating cells for 5 days and still observed only a slight increase in NOTCH3 protein expression (Fig. S3D). Thus, MYC is not sufficient in this context to transcriptionally induce $\mathrm{NOTCH} 3$, although it may have some slight effect on NOTCH3 protein expression.
A

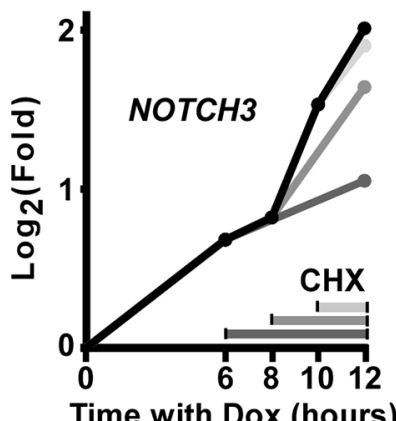

D

PPrEC-TetON-MKK6(CA)

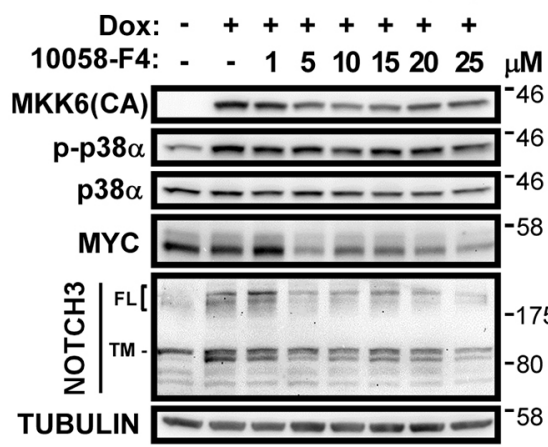

B

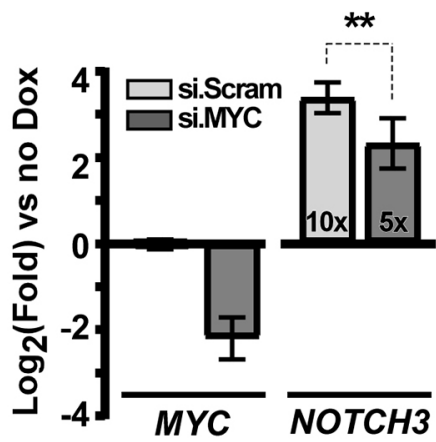

$\mathbf{E}$

iPrEC-TetON-MKK6(CA)

Dox: -++++++

JQ1: - $\quad$ - $1101005001,000 \mathrm{nM}$

MKK6(CA) ---

p-p38

p38 $\alpha-1-1-1-1-1$

MYc $=-1-2=-48$

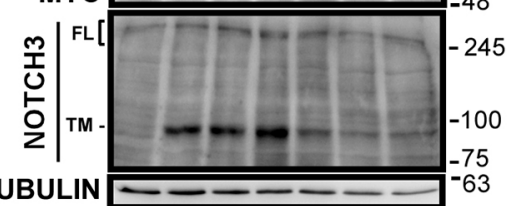

C

Pre $\mid$ siScram siMYC

T-fect 0 oh 6h 12h 0h 6h 12h Dox

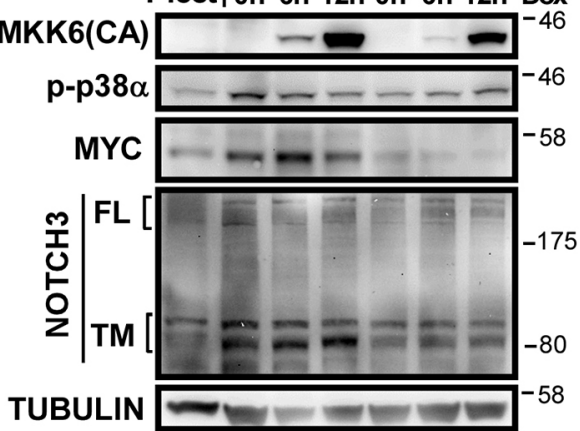

$\mathbf{F}$

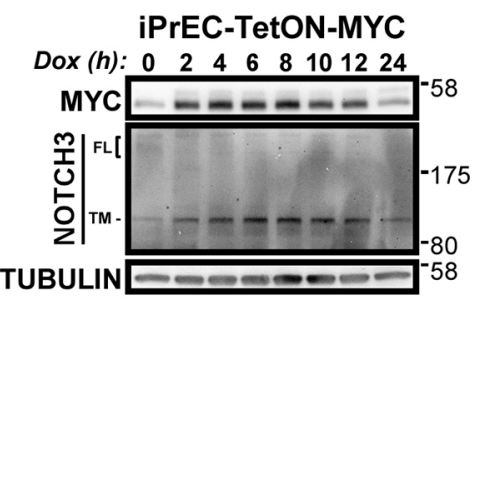

Fig. 4. MYC is an intermediate for p38-MAPK induction of NOTCH3. (A) iPrEC-TetON-MKK6(CA) cells were induced with Dox for a total of $12 \mathrm{~h}$ with cyclohexamide $(\mathrm{CHX})$ added at 6,8 or $10 \mathrm{~h}$. NOTCH3 mRNA was measured by qRT-PCR. Data were normalized to the value at $0 \mathrm{~h}$. (B) iPrEC-TetON-MKK6(CA) cells were transfected with siMYC or siScram for $24 \mathrm{~h}$, then treated with Dox for $12 \mathrm{~h}$ and analyzed by qRT-PCR. Data were normalized to non-transfected untreated controls. Graph shows mean \pm s.d. of biological triplicates. Numbers in bars indicate fold change. ${ }^{* *} P \leq 0.01$. (C) Same experiment set-up as B, except lysates were harvested at 0,6 or $12 \mathrm{~h}$ after Dox treatment and used for immunoblotting. FL, full length; TM, transmembrane. (D) iPrEC-TetON-MKK6(CA) cells were treated for $16 \mathrm{~h}$ with Dox plus DMSO or increasing doses of MYC inhibitor 10058-F4. Protein was analyzed by immunoblotting. (E) Same experimental set-up as D, but using a different MYC inhibitor, JQ1. (F) iPrECs expressing Dox-inducible MYC (iPrEC-TetON-MYC) were treated with Dox for 0-24 h and analyzed by immunoblotting. 


\section{NOTCH3 is transcriptionally regulated via a MYC-dependent} enhancer

The NOTCH3 $2 \mathrm{~kb}$ upstream proximal promoter contains a $\mathrm{CpG}$ island and no TATA sequence (Kent et al., 2002). The $2 \mathrm{~kb}$ region of the NOTCH3 promoter was not sufficient to induce a luciferase reporter after 6 days of differentiation (Fig. 5A), a time when endogenous NOTCH3 was elevated over 16-fold. We used two approaches to identify candidate enhancer regions. First, we labeled newly initiated transcripts at the NOTCH3 transcriptional start site and enhancer elements by using BruUV-Seq (Magnuson et al., 2015). Dox induction in iPrEC-TetON-MKK6(CA) cells dramatically increased $\mathrm{NOTCH} 3$ reads from the coding $(-)$ strand accumulating near the transcription start site (Fig. 5B). Strikingly, there was also a peak of reads from the non-coding $(+)$ strand within the second intron, a locus previously reported to contain a NOTCH3 enhancer (Gagan et al., 2012; Romano et al., 2012). The gene for MKK6 (MAP2K6) served as a positive control; it was induced only upon Dox treatment and with reads mapping only to the exons generated from the cDNA construct (Fig. S4A). Other controls included CALB1 and TRIM22, which were increased and decreased, respectively, upon MKK6 induction (Fig. S4A).

Our second approach used a combination of DNase hypersensitivity, histone acetylation and methylation patterns
(H3K27Ac+H3K4me1/2), and ChIP-seq data from ENCODE to identify potential enhancer elements (The ENCODE Project Consortium, 2012; Kent et al., 2002). Five different elements were cloned into a pNL1.1-miniTK luciferase reporter (Fig. S4B). En2.1, En2.2 and the NOTCH3 promoter showed no induction by Dox in the MKK6(CA) model (Fig. 5C). However, two elements (En1 and En3) were upregulated by 5- and 3-fold, respectively. En1 is $\sim 10 \mathrm{~kb}$ upstream while En3 is in the second intron and corresponds to the site with bidirectional transcripts identified by BruUV-seq. A deletion in En1, $\Delta 1-360$, that eliminated most of the predicted MYC-binding sites (Fig. S4C) completely ablated the ability of the En1 reporter to be induced by MKK6(CA) (Fig. 5D). A small En3 deletion, $\Delta 1-350$, that removed two-thirds of the predicted MYC sites did not significantly decrease expression of the reporter while a larger deletion, $\Delta 1-655$, that removed all three predicted MYC sites significantly blocked induction (Fig. 5E).

To further determine whether MYC is required for induction of these enhancer elements, MKK6(CA) cells were induced in the presence of the MYC inhibitor 10058-F4. Both En1 and En3 $(\Delta 1-350)$ (the core En3 responsive element) were sensitive to MYC inhibition (Fig. 5F). Induction mediated by En1 was partially decreased (2.7- vs 4.5-fold) while En $3(\Delta 1-350)$ induction was more thoroughly blocked (0.7- vs 1.7-fold). Thus, both En1 and En3 are
A

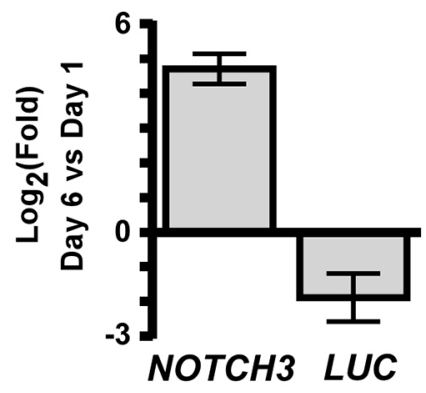

C

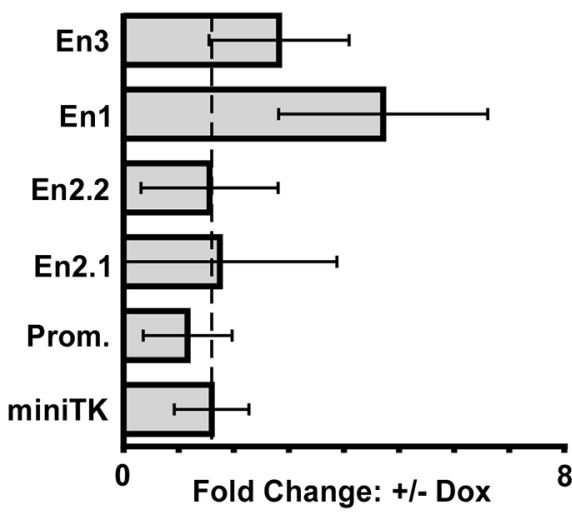

B

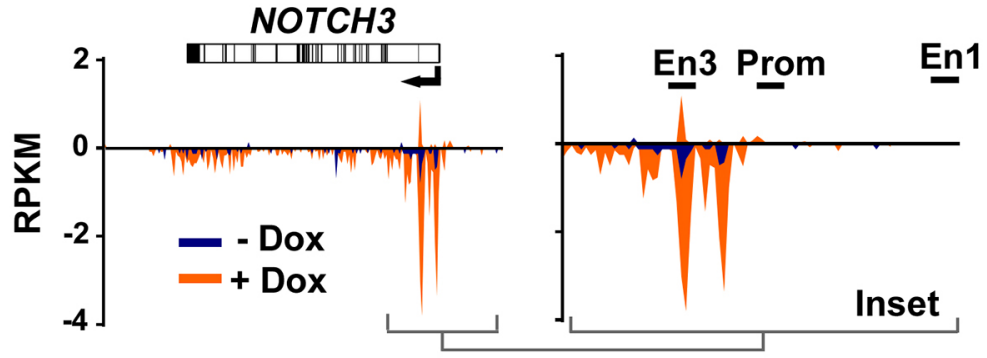

D

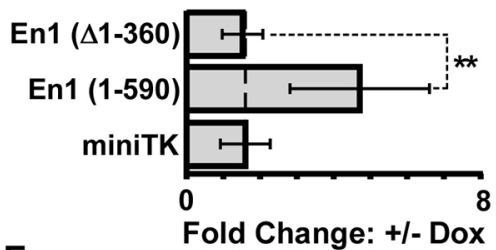

$\mathbf{F}$

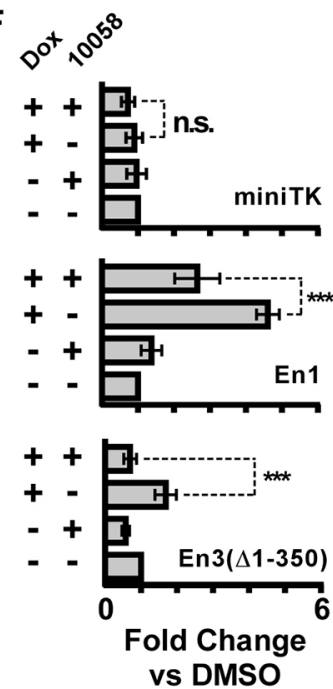

Fig. 5. NOTCH3 transcription requires a MYC-driven enhancer element. (A) iPrECs were stably transfected with a luciferase reporter driven by 2 kb of NOTCH3 upstream sequence. A stable pool was differentiated for 1 or 6 days, and analyzed by qRT-PCR. Data were standardized to $18 S$ and ACTB and normalized to the value at day 1 . Graph shows mean \pm s.d. of biological triplicates. (B) iPrEC-TetON-MKK6(CA) cells were treated with or without Dox for $10 \mathrm{~h}$ and processed for BruUV-Seq. The $y$-axis is RPKM (reads per kilobase of transcript per million mapped reads). Plus-strand reads are given above the $x$-axis, minusstrand reads are below the x-axis. Blue, without Dox; orange, with Dox. A NOTCH3 gene diagram shows orientation (arrow) and exons (black lines). (C-F) iPrECTetON-MKK6(CA) cells were transfected with indicated luciferase reporter constructs (see Fig. S4B), split, and then treated with or without Dox for $16 \mathrm{~h}$. Graphs show mean $\pm 95 \%$ c.i. In C, $n=8$ from two experiments; in D,E $n=6$ from one experiment. (F) In addition to Dox, cells were treated with DMSO or $10058-F 4$ (20 $\mu M$ ). $n=8$ from one experiment, normalized to without Dox (DMSO control). ${ }^{*} P \leq 0.05 ;{ }^{* *} P \leq 0.01$; ${ }^{* *} P \leq 0.001$; n.s., not significant. 
sensitive to MYC inhibition and both contain MYC-binding sites, which when deleted significantly reduced reporter induction in response to MKK6(CA).

\section{NOTCH3 expression is controlled by mRNA stability}

NOTCH3 contains an AU-rich element in its 3' UTR and p38MAPK is known to regulate RNA-binding proteins (Cuadrado and Nebreda, 2010). Actinomycin D was used to halt transcription, and measurements of mRNA decay were taken at nine time points (Harrold et al., 1991) at day 1 and day 4 of differentiation (Fig. 6A; Table 1). The $M Y C$ half-life, of $0.8 \mathrm{~h}$, was similar to that found in previous reports (Herrick and Ross, 1994). MYC and NOTCH1 halflives remained essentially the same at day $4(P>0.2)$. However, NOTCH3 mRNA half-life nearly doubled (11.5 vs $5.9 \mathrm{~h})$, along with an 8.5-fold increase in total mRNA levels. We similarly compared iPrEC-TetON-MKK6(CA) cells stimulated with Dox for $16 \mathrm{~h}$ to non-Dox-treated cells (Fig. 6B; Table 2). Both NOTCH1 and NOTCH3 mRNA half-lives more than doubled: 3.3 to $8.8 \mathrm{~h}$ for NOTCH1, and 7.6 to $17.6 \mathrm{~h}$ for NOTCH3. However, the overall mRNA level of NOTCH1 decreased $\sim 4$-fold while NOTCH3 increased $\sim 9$-fold (Table 2). Thus, differentiation and acute p38MAPK activation both lead to increased NOTCH3 mRNA half-life, indicating that $\mathrm{NOTCH} 3$ is regulated post-transcriptionally through mRNA stabilization.

\section{DISCUSSION}

\section{Differential regulation of NOTCH1 and NOTCH3 during} differentiation

NOTCH1 expression has been reported to primarily be present in basal cells of mouse and human prostate, while NOTCH3 has been reported (with some disagreement) to be more luminal (Pedrosa et al., 2016; Shou et al., 2001; Valdez et al., 2012). We detected abundant NOTCH1 and NOTCH2 and very low NOTCH3 in undifferentiated human basal cells. NOTCH4 protein was detectable but at a very low level and did not increase during differentiation (not shown). Owing to their dynamic regulation during differentiation, we focused on NOTCH1 and NOTCH3. We observed a dramatic induction of NOTCH3 mRNA and protein during differentiation, which coincided with the appearance of suprabasal cells. Therefore, NOTCH3 appears to be a primary driver of luminal cell differentiation, while NOTCH1 serves its previously described role in maintaining the basal population (Pedrosa et al., 2016; Shou et al., 2001; Valdez et al., 2012).

Previous studies have shown that low- $\mathrm{Ca}^{2+}$ medium, such as the KSFM in which we culture our cells, selects for basal transitamplifying prostate epithelial cells and promotes their survival via constitutive activation of NOTCH1 (Dalrymple et al., 2005; Litvinov et al., 2006). However, inhibition or knockdown of NOTCH1 or NOTCH3 did not affect basal cell survival in our assays (Fig. S2E). In the previous studies, constitutive NOTCH1 signaling was most important in subconfluent cultures. We only inhibited NOTCH in completely confluent cells, which may account for the observed differences.

The function of NOTCH3 has been controversial, but recent reports show that it drives luminal differentiation of airway basal cells and mammary epithelium (Baeten and Lilly, 2015; Bhat et al., 2016; Gomi et al., 2015; Mori et al., 2015; Ohashi et al., 2010). Moreover, of the four NOTCH receptors only NOTCH3 is sufficient to drive hepatocyte differentiation in embryonic mouse liver cells (Ortica et al., 2014). Though NOTCH1 seems to drive prostate basal cell commitment, our data supports the idea that $\mathrm{NOTCH} 3$ is required to generate the suprabasal cell layer required for prostate luminal cell differentiation.

\section{Transcriptional regulation of NOTCH3 by p38-MAPK}

Part of the mechanistic insight from this work demonstrates that p38-MAPK can regulate NOTCH3 transcription in part via MYC. Although a relationship between p38-MAPK and NOTCH has previously been suggested, mechanistic details were not clearly established (Brown et al., 2009; Gonsalves and Weisblat, 2007; Kiec-Wilk et al., 2010; Park et al., 2009). We found that the full ability of p38-MAPK to induce NOTCH3 is dependent on MYC. We previously demonstrated $\mathrm{MYC}$ is required for PrEC differentiation (Berger et al., 2014; Marderosian et al., 2006). Thus, NOTCH3 appears to be one of the MYC targets downstream of p38-MAPK. MYC has typically been considered a downstream target of NOTCH (Weng et al., 2006), whereas we found that it is upstream of $\mathrm{NOTCH}$. Although $\mathrm{MYC}$ was required for full NOTCH3 induction, blocking its activity did not fully block NOTCH3 induction suggesting that there are likely other factors involved. Furthermore, overexpression of MYC was not sufficient to induce NOTCH3 mRNA. Thus, p38-MAPK is likely activating
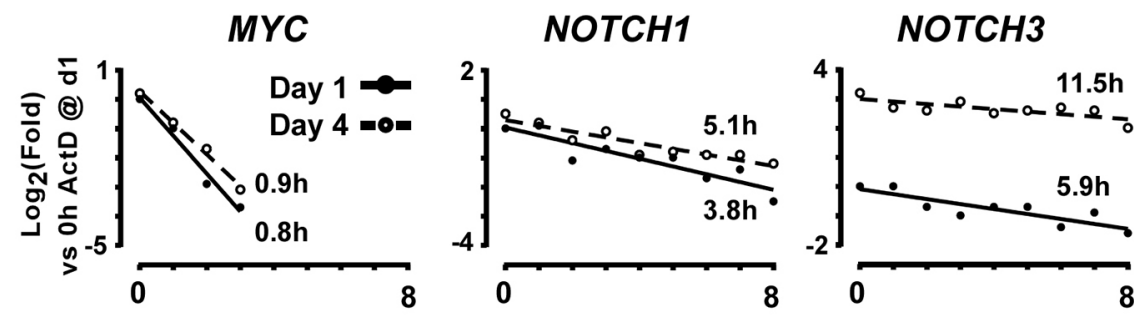

B

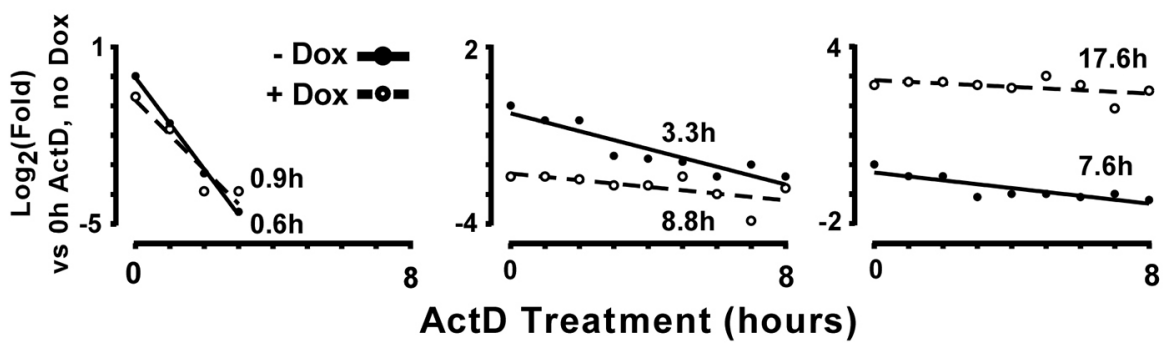

Fig. 6. p38-MAPK upregulates NOTCH3 mRNA stability. (A) iPrECs were differentiated for 1 or 4 days and at each time treated with actinomycin D (ActD) for 0-8 h. RNA was harvested for qRT-PCR analysis. Samples were standardized to $18 \mathrm{~S}$ rRNA and normalized to the day 1, 0 h sample. (B) Same as in A except using the iPrEC-TetON-MKK6(CA) model. Cells were treated with or without Dox for $16 \mathrm{~h}$ prior to ActD treatment. Samples were normalized to the $0 \mathrm{~h}$ sample without Dox. The numbers given are the calculated half-life in hours. 
Table 1. Day 4 vs Day 1 mRNA half-life calculations

\begin{tabular}{lllllll}
\hline & & $\begin{array}{c}\text { Line equation } \\
(y=m x+b)\end{array}$ & $r^{2}$ & $\begin{array}{l}\text { Half-life } \\
(1 / \mathrm{m})\end{array}$ & $\begin{array}{l}P \text {-value } \\
(\mathrm{m} 1 \mathrm{vs} \mathrm{m} 2)\end{array}$ & $\begin{array}{l}\text { Overall } \\
\text { expression }\end{array}$ \\
\hline MYC & Day1 & $y=-1.30 x+0.05$ & 0.98 & $0.8 \mathrm{~h}$ & 0.25 & +1.2 fold \\
& Day4 & $y=-1.08 x+0.27$ & 0.99 & $0.9 \mathrm{~h}$ & & \\
\multirow{2}{*}{ NOTCH1 } & Day1 & $y=-0.267 x+0.03$ & 0.82 & $3.8 \mathrm{~h}$ & 0.23 & +1.2 fold \\
& Day4 & $y=-0.197 x+0.29$ & 0.85 & $5.1 \mathrm{~h}$ & & \\
\multirow{2}{*}{ NOTCH33 } & Day1 & $y=-0.170 x-0.10$ & 0.74 & $5.9 \mathrm{~h}$ & 0.11 & +8.5 fold \\
& Day4 & $y=-0.0867 x+2.99$ & 0.55 & $11.5 \mathrm{~h}$ & &
\end{tabular}

$\mathrm{r}^{2}$ values indicate how well the nine data points fit each linear regression line. $P$-values compare slopes between lines using ANCOVA analysis.

additional unidentified factors that are also required for $\mathrm{NOTCH3}$ mRNA induction.

\section{Identification and validation of a novel NOTCH3 enhancer}

We investigated potential regulatory regions of the NOTCH3 gene and found two elements capable of inducing a luciferase reporter upon MKK6(CA) induction that are sensitive to MYC inhibition. One element lies $\sim 10 \mathrm{~kb}$ upstream, denoted En1, and has not previously been linked to NOTCH3. A $5^{\prime}$ deletion that eliminates most of the predicted MYC-binding sites in En1 severely compromises its induction; however, it is only partially sensitive to inhibition of MYC. Thus, there are likely to be other factors that cooperate with MYC to fully activate this enhancer. A second element, En3, lies in a previously implicated locus within the second intron (Gagan et al., 2012; Romano et al., 2012). Our report is the first to show functional validation of En3 in human cells. Furthermore, we identified bi-directional eRNA from En3 upon p38-MAPK stimulation, as measured by BruUV-Seq (Kim et al., 2010; Lam et al., 2014; Magnuson et al., 2015). A small deletion (En3 $\Delta 1-350)$ that removed two-thirds of the predicted MYC sites retained reporter activity, thus narrowing down the core regulatory region. Likewise, a second larger deletion (En3 $\Delta 1-655)$ that removed all the predicted MYC-binding sites greatly diminished induction of the reporter. Both elements contain numerous other potential transcription factor-binding sites (The ENCODE Project Consortium, 2012; Mathelier et al., 2016) that may be required for NOTCH3 to cooperate with MYC. Further detailed analysis will be required to completely define all possible mechanisms of $\mathrm{NOTCH} 3$ transcriptional regulation.

\section{NOTCH3 regulation via mRNA stability}

We also demonstrate that $N O T C H 3$ is post-transcriptionally regulated through mRNA stability during differentiation mediated by p38-MAPK. NOTCH1 expression is affected by RNA stability, which is known to be modulated through AU-rich elements in its $3^{\prime}$ untranslated region (UTR) and by p38-MAPK (Cisneros et al., 2008; Gonsalves and Weisblat, 2007). p38-MAPK regulates mRNA stability through phosphorylation of mRNA-binding proteins

Table 2. MKK6(CA) mRNA half-life calculations

\begin{tabular}{lllllll}
\hline & & $\begin{array}{c}\text { Line equation } \\
(y=m x+b)\end{array}$ & $\mathrm{r}^{2}$ & $\begin{array}{c}\text { Half-life } \\
(1 / \mathrm{m})\end{array}$ & $\begin{array}{l}P \text {-value } \\
(\mathrm{m} 1 \mathrm{vs} \mathrm{m} 2)\end{array}$ & $\begin{array}{l}\text { Overall } \\
\text { expression }\end{array}$ \\
\hline MYC & - Dox & $y=-1.55 x-0.05$ & 1.00 & $0.6 \mathrm{~h}$ & 0.50 & +1.7 fold \\
& + Dox & $y=-1.17 x-0.82$ & 0.90 & $0.9 \mathrm{~h}$ & & \\
NOTCH1 & - Dox & $y=-0.302 x-0.26$ & 0.85 & $3.3 \mathrm{~h}$ & 0.02 & -4.1 fold \\
& + Dox & $y=-0.113 x-2.30$ & 0.42 & $8.8 \mathrm{~h}$ & & \\
NOTCH3 & - Dox & $y=-0.132 x-0.27$ & 0.73 & $7.6 \mathrm{~h}$ & 0.14 & +8.8 fold \\
& + +Dox & $y=-0.057 x+2.86$ & 0.25 & $17.6 \mathrm{~h}$ & & \\
\end{tabular}

$\mathrm{r}^{2}$ values indicate how well the nine data points fit each linear regression line. $P$-values compare slopes between lines using ANCOVA analysis.
(Cuadrado and Nebreda, 2010). NOTCH3 also has predicted AUrich elements in its 3' UTR (Gruber et al., 2011). Interestingly, p38MAPK activation via MKK6(CA) for $16 \mathrm{~h}$ increased both NOTCH1 and NOTCH3 mRNA half-life, but only NOTCH3 stability was increased after 6 days of differentiation. This may reflect differences in the extent of p38-MAPK activation in the two models or may suggest that other modes of stabilization are involved. There are reports of post-transcriptional NOTCH regulation by micro (mi) RNAs, which may also contribute to long-term stability (Furukawa et al., 2013; Gagan et al., 2012; Liu et al., 2015).

We also found that MYC enhances NOTCH3 expression independently of mRNA. For instance, it took $20 \mu \mathrm{M}$ of MYC inhibitor (10058-F4) to suppress NOTCH3 mRNA expression, yet there were effects on NOTCH3 protein at $5 \mu \mathrm{M}$. Similarly, overexpression of MYC did not alter NOTCH3 mRNA, but it did increase NOTCH3 protein, suggesting that there may be a mechanism for stabilizing NOTCH3 protein or increasing its translation rate. In addition, shRNA against NOTCH3 resulted in partial loss of NOTCH1 protein, but not mRNA. Thus, there are several mechanisms that regulate both NOTCH1 and NOTCH3 during luminal cell differentiation, and further research will be required to define them all.

\section{Role for AR in differentiation}

One of the key roles for AR in normal luminal differentiation is to inhibit proliferation, which is the opposite of its role in tumors. Previous reports have shown that AR overexpression in basal PrECs can induce growth arrest and that this requires $\mathrm{AR}$ (in cooperation with $\beta$-catenin/TCF-4), which transcriptionally represses MYC (Antony et al., 2014; Vander Griend et al., 2014). This is opposite to what is seen in tumors, where AR can drive MYC expression (Antony et al., 2014). Our data showed that p38-MAPK can upregulate MYC expression, which is transient in our differentiation model. Although we have not investigated it, AR may help suppress MYC expression once the suprabasal layer is established. Likewise, it may be that full luminal commitment and increased AR activity may provide a brake for NOTCH3 induction by antagonizing MYC.

\section{Day 8 is a critical transition point in differentiation}

Temporal regulation of $\mathrm{NOTCH} 3$ throughout differentiation is dynamic. We observed two phases of NOTCH3 mRNA induction: an early steady increase up to day 8 (day 4 in primary cells) followed by a more dramatic increase. Considering that $N O T C H 3$ mRNA is stabilized by day 6 , it could be that early upregulation is less dependent on transcriptional mechanisms and more on message stability. The suprabasal layer is visible at around day 8 , coinciding with induction of downstream target $H E S$ and $H E Y$ genes. Additionally, it is at this transition point that p38-MAPK and MYC are activated. Thus, robust transcriptional induction of NOTCH3 appears to peak at around this time and may drive the secondary phase of NOTCH3 induction. It is also at this time that NOTCH1 mRNA begins to increase following an initial dip. Thus, day 8 is a key point for NOTCH1 and NOTCH3 induction and cell commitment to the luminal transition.

\section{Potential downstream effects of NOTCH activity}

The direct effectors of NOTCH signaling include the canonical $H E S$ and $H E Y$ transcriptional repressor family. Indeed, we observed differential induction of several family members during differentiation. In ongoing studies, we are determining which of these are critical for luminal cell differentiation. Previous findings have reported that AR and GATA cooperate 
to regulate a set of target genes, and HEY transcriptional repressors can prevent GATA-mediated induction of AR target genes (Belandia et al., 2005; Fischer et al., 2005; Litvinov et al., 2006; Xiao et al., 2016). This would support downstream HEY activity in maintaining a basal commitment. With the $\mathrm{NOTCH}$ pathway, timing and dose are critical. Our attempts to drive differentiation with inducible NOTCH ICD (NICD) constructs led to cell stress and death within 24-48 h (data not shown). It may be that a low or moderate amount of $\mathrm{NOTCH}$ activity is needed for survival and initial differentiation but too much activity can block terminal differentiation. Whether the functional role of $\mathrm{NOTCH} 3$ is via $\mathrm{HES}$ and HEY or non-canonical downstream targets will require further investigation.

Some of the reported non-canonical NOTCH targets include PTEN and CDH1 (also known as E-cadherin), both of which are critical for luminal cell survival (Bertrand et al., 2014; Lamb et al., 2010). Furthermore, NOTCH downregulates adhesion genes, including integrins such as $\beta 4$, which is required for basal cell detachment from the extracellular matrix (Cress et al., 1995; Mazzone et al., 2010; Nguyen et al., 2006). We also see loss of integrin expression during differentiation. There are also reports that NOTCH can upregulate MKP1 (also known as DUSP1), a phosphatase that targets p38-MAPK, thus providing a potential feedback mechanism in terminally differentiated cells to balance p38-MAPK activity (Gagan et al., 2012; Yoshida et al., 2014). The balance of downstream NOTCH targets (both canonical and noncanonical) could help explain the conflicting roles for the pathway in promoting both basal and luminal commitments.

Previous studies suggested that the ICD from NOTCH3 is a weaker activator than other NICDs (Beatus et al., 1999; Ong et al., 2006). However, our findings and other recent reports have begun to reveal novel signaling effects and preferential targets for NOTCH3 (Baeten and Lilly, 2015; Cui et al., 2013; Wang et al., 2016). As it stands, NOTCH3 appears to be unique among the receptors. Further research will be needed to validate which downstream NOTCH3-specific targets are most relevant to luminal cell differentiation.

\section{Conclusion}

In this study, we report on a novel mechanism for crosstalk between p38-MAPK, MYC and NOTCH. Moreover, we identify two distinct regulatory mechanisms for NOTCH3 in the prostate: a coordination of elevated mRNA stability and increased transcription from multiple enhancers. These findings provide a better understanding for how these differentiation pathways are connected in normal prostate epithelium and opens the door to investigating how their dysregulation may impact prostate cancer development and progression.

\section{MATERIALS AND METHODS \\ Cell culture}

Primary and immortalized PrECs (Berger et al., 2014) were grown in KSFM medium (Gibco) plus penicillin-streptomycin at 30 units $/ \mathrm{ml}$ (Gibco). Differentiation was induced as previously reported with $2.5 \mathrm{ng} / \mathrm{ml}$ recombinant KGF (Cell Sciences) and 1-10 nM R1881 (Perkin Elmer) (10 $\mathrm{nM}$ unless otherwise specified) with fresh medium added every $24 \mathrm{~h}$ (Lamb et al., 2010). Suprabasal layer separation was achieved by using $\mathrm{Ca}^{2+}$ and $\mathrm{Mg}^{2+}$-free PBS with $1 \mathrm{mM}$ EDTA as previously described (Lamb et al., 2010). HEK 293FT cells were used for lentivirus production (ViraPower, Invitrogen) and grown in Dulbecco's modified Eagle's medium (DMEM; 11995, Gibco) with $10 \%$ fetal bovine serum (FBS; Gemini) and $2 \mathrm{mM}$ L-glutamine (Gibco). Cell lines were tested via a MycoAlert PLUS kit (Lonza) and confirmed to be mycoplasma free.

\section{Molecular cloning and stable cell line construction}

Immortalized PrECs (iPrECs) were engineered with Dox-inducible shRNAs using the EZ-Tet-pLKO-Puro and EZ-Tet-pLKO-Hygro vectors (Addgene plasmids 85966, 85972) (Frank et al., 2017). shRNA sequences are listed in Table S1. Expression cDNAs were subcloned, via PCR with Q5 polymerase (NEB), into the pENTR3C gateway vector (Invitrogen) between the SalI and NotI sites and then recombined with LR Clonase II (Invitrogen) into pLentiCMV/TO-Puro-DEST (Addgene plasmid 17293) (Campeau et al., 2009). The constitutively active MKK6 mutant (MKK6-DD) was a gift from Angel Nebreda (Oncology Unit, Institute for Research in Biomedicine, Spain) (Alonso et al., 2000). The MYC cDNA, subcloned from pBabe-Myc, was a gift from Beatrice Knudsen (Biomedical Sciences and Pathology, Cedars Sinai, USA). TetR lines were established by using pLenti-CMV-TetR-Blast (Addgene plasmid 17492) (Campeau et al., 2009). iPrECs antibiotic selection doses were as follows: $50 \mu \mathrm{g} / \mathrm{ml}$ hygromycin, $5 \mu \mathrm{g} / \mathrm{ml}$ blasticidin and $2 \mu \mathrm{g} / \mathrm{ml}$ puromycin. Doxycycline (Sigma) was used at $50 \mathrm{ng} / \mathrm{ml}$ to induce shRNAs and 2-10 $\mathrm{ng} / \mathrm{ml}$ to induce cDNA expression.

\section{siRNA and inhibitors}

A mixed siRNA pool against $M Y C$ and non-targeting siRNA (siScram) were purchased from Origene (SR303025). Cells were transfected by using siLentfect reagent (Bio-Rad). Cyclohexamide was used at $10 \mu \mathrm{g} / \mathrm{ml}$, and actinomycin D at $5 \mu \mathrm{g} / \mathrm{ml}$ (Calbiochem). SB202190, BIRB796/ Doramapimod, 10058-F4, JQ-1, BrdU and staurosporine were purchased from Cayman Chemical. RO4929097 was purchased from Apex Bio.

\section{Immunoblotting}

Cell lysates were prepared in RIPA as previously described (Edick et al., 2007). Protein loading was standardized by use of the BCA assay (Pierce). $20-50 \mu \mathrm{g}$ of denatured protein was run on Novex SDS polyacrylamide Trisglycine gels (Life Technologies) and transferred onto PVDF membrane (Fisher). Chemiluminescence was used to image blots with a Bio-Rad Chemi-Doc imaging system with CCD camera. The quantification shown in Fig. S1A,B was performed with ImageJ software. Data were first normalized to tubulin, then to day 1 ' $\mathrm{i}$ ' samples and plotted as mean \pm s.d. $P$-values were determined by paired one-way ANOVA with Turkey's multiple testing correction. Antibodies are listed in Table S2. The protein ladder was from Cell Signaling Technology (7720) or GoldBio (P007).

\section{qRT-PCR}

RNA was harvested and extracted with Trizol following the manufacturer's protocol (Invitrogen). cDNA was synthesized with M-MuLV reverse transcriptase (NEB) using a 4:1 mix of poly-d(16)T and random hexamer primers. qRT-PCR was performed using SYBR Green Master Mix (Roche) and an ABI 7500 thermal cycler (Applied Biosystems). Data were standardized to $18 S$ plus GAPDH unless otherwise stated and were normalized $(\triangle \Delta C T)$ and plotted as $\log _{2}($ Fold $)$. Primers were synthesized by Integrated DNA Technologies. Primers are in Table S3.

\section{Immunostaining}

Cells were fixed, permeabilized, and stained as previously described (Berger et al., 2014). Antibodies against ITG $\alpha 6(555734, \mathrm{BD})$ and AR (sc-815, Santa Cruz Biotechnology) were used at 1:200 dilution. Suprabasal coverage of the underlying basal layer was determined by tracing the clusters that were both $\mathrm{AR}^{+}$and $\mathrm{ITG} \alpha 6^{-}$by hand using ImageJ software, and calculating the percentage area of suprabasal regions versus total image area. Three fields of view were measured for each condition. For propidum iodide staining, cells were fixed with $4 \%$ paraformaldahyde, treated with $100 \mathrm{ng} / \mathrm{ml}$ RNaseA (Thermo) for $10 \mathrm{~min}$, then stained with $100 \mathrm{ng} / \mathrm{ml}$ propidum iodide (Sigma) for $5 \mathrm{~min}$. Nuclei were stained with $10 \mu \mathrm{g} / \mathrm{ml}$ Hoescht 33258 (Sigma) for $10 \mathrm{~min}$. Epifluorescence microscopy was performed on a Nikon TE300 using Nikon Elements software (v4.11.00). Fig. 2E was captured on a DeltaVision (GE) epifluorescence scope with SoftWoRx software, with processing by deconvolution and maximum intensity projection from a $z$-stack capture.

\section{Luciferase assay and constructs}

Putative NOTCH3 regulatory elements were PCR subcloned from the RP11937H1 BAC library (Life Technologies) using Q5 or LongAmp polymerase 
(NEB). The NOTCH3 $2 \mathrm{~kb}$ promoter element was ligated into pGL4.15Hygro (Promega). Candidate regulatory elements were ligated into pNL1.1 (Promega) after first cloning in a miniTK promoter at the HindIII site. Deletion mutants were made using the QuickChange II Mutagenesis kit (200524, Stratagene). Cloning primers, miniTK sequence, and mutagenesis primers are in Table S4.

En1 and En3 maps in Fig. S4C were generated using SnapGene and modified with Canvas software. MYC-binding sites were determined by using the JASPAR online database (http://jaspar.genereg.net/) (Mathelier et al., 2016), with a threshold of $80 \%$ using the MA0147.2 matrix model for MYC-binding sites (Chen et al., 2008).

Luciferase assays were performed by using the NanoGlo kit (Promega) and a Synergy Neo II (Bio Tek) plate reader with Gen5 software (v2.04). Cells were transfected as a pool with XtremeGeneHP reagent (Roche) and then split for different treatments. pNL1.1-miniTK served as the negative control. Luciferase assays were run $16 \mathrm{~h}$ after Dox treatment and $48 \mathrm{~h}$ after transfection.

\section{mRNA half-life measurement}

Cells were treated with $5 \mu \mathrm{g} / \mathrm{ml}$ Actinomycin D for $0-8 \mathrm{~h}$. RNA and cDNA were prepared as described above. Data were standardized to $18 \mathrm{~S}$ rRNA and normalized as $\Delta \Delta \mathrm{CT}$ values versus the Day 1 or 'no Dox' samples at $0 \mathrm{~h} .18 \mathrm{~S}$ rRNA has a very long half-life (1-7 days) and thus is suitable for standardization (Defoiche et al., 2009). Linear regression curves, line equations, $\mathrm{r}^{2}$ values, and $P$-values were calculated with GraphPad PRISM software. Half-life was calculated as $1 / \mathrm{m}$, where $\mathrm{m}$ is the slope. Overall expression change was calculated as $2^{(b 2-b 1)}$, where $b=y$-intercept; $b 1$ is intercept 1 and $\mathrm{b} 2$ is intercept 2 . AU-rich elements were identified using the ARE site (v1) online tool (http://nibiru.tbi.univie.ac.at/cgi-bin/AREsite/ AREsite.cgi) (Gruber et al., 2011).

\section{BruUV-Seq}

iPrEC-TetON-MKK6(CA) cells were treated with $5 \mathrm{ng} / \mathrm{ml}$ Dox for $10 \mathrm{~h}$ or left untreated, then exposed to UV $\left(100 \mathrm{~J} / \mathrm{m}^{2}\right)$ using a Stratalinker UV Crosslinker 1800 (Stratagene) and labeled with $2 \mathrm{mM}$ 5-bromo-deoxyuridine (sc-256904, Santa Cruz Biotechnology) for $30 \mathrm{~min}$ before washing with PBS and collecting RNA with Trizol (Life Technologies). BrU isolation, library prep, sequencing and mapping was performed as previously described (AndradeLima et al., 2015; Paulsen et al., 2014). Data were exported (bin size $=300$ bp) and graphed using GraphPad PRISM software.

\section{Statistical analysis}

Unless otherwise specified, $P$-values were calculated using paired, one-tailed $t$-tests on biological triplicates, with $* P \leq 0.05,{ }^{*} * P \leq 0.01$, *** $P \leq 0.001$ and $\mathrm{n}$. s., not significant $(P>0.05)$. For Tables 1 and $2, P$-values were calculated by ANCOVA analysis using PRISM GraphPad software. Fig. 5D,E used oneway ANOVA with Greenhouse-Geisser correction. Fig. 5F used two-way ANOVA with Turkey's multiple testing correction.

\section{Acknowledgements}

We acknowledge Mary Winn and the VARI Bioinformatics and Biostatistics Core for assistance with statistical and RNA-Seq analysis. We thank Michelle Paulsen for technical consolation on BruUV-Seq. Additional thanks to the VARI Program for Skeletal Disease and Tumor Metastasis for feedback and suggestions. The TetON cDNA and TetR vectors were gifts from Eric Campeau, the MMK6(CA) (MKK6-DD) construct was a gift from Dr. Angel Nebreda, and pBabe-MYC a gift from Dr. Beatrice Knudsen.

\section{Competing interests}

The authors declare no competing or financial interests.

\section{Author contributions}

Conceptualization: S.B.F., M.L., C.K.M.; Methodology: S.B.F., P.L.B., M.L.; Software: M.L.; Validation: S.B.F., P.L.B.; Formal analysis: S.B.F., M.L., C.K.M.; Investigation: P.L.B.; Resources: C.K.M.; Data curation: P.L.B.; Writing - original draft: S.B.F., C.K.M.; Writing - review \& editing: S.B.F., C.K.M.; Supervision: C.K.M.; Project administration: C.K.M.; Funding acquisition: C.K.M.

\section{Funding}

These studies were supported by funding from the U.S. Department of Defense Prostate Cancer Research Program W81XWH-14-1-0479 (to C.K.M., S.B.F. and
P.L.B.), Association for International Cancer Research (Worldwide Cancer Research) (11-0082 to S.B.F.), the Van Andel Research Institute and the University of Arizona.

\section{Supplementary information}

Supplementary information available online at

http://jcs.biologists.org/lookup/doi/10.1242/jcs.197152.supplemental

\section{References}

Alonso, G., Ambrosino, C., Jones, M. and Nebreda, A. R. (2000). Differential activation of p38 mitogen-activated protein kinase isoforms depending on signal strength. J. Biol. Chem. 275, 40641-40648.

Andrade-Lima, L. C., Veloso, A., Paulsen, M. T., Menck, C. F. M. and Ljungman, M. (2015). DNA repair and recovery of RNA synthesis following exposure to ultraviolet light are delayed in long genes. Nucleic Acids Res. 43, 2744-2756.

Antony, L., van der Schoor, F., Dalrymple, S. L. and Isaacs, J. T. (2014) Androgen receptor (AR) suppresses normal human prostate epithelial cell proliferation via AR/beta-catenin/TCF-4 complex inhibition of c-MYC transcription. Prostate 74, 1118-1131.

Baeten, J. T. and Lilly, B. (2015). Differential regulation of $\mathrm{NOTCH} 2$ and $\mathrm{NOTCH} 3$ contribute to their unique functions in vascular smooth muscle cells. J. Biol. Chem 290, 16226-16237

Beatus, P., Lundkvist, J., Oberg, C. and Lendahl, U. (1999). The notch 3 intracellular domain represses notch 1-mediated activation through Hairy/ Enhancer of split (HES) promoters. Development 126, 3925-3935.

Belandia, B., Powell, S. M., Garcia-Pedrero, J. M., Walker, M. M., Bevan, C. L. and Parker, M. G. (2005). Hey1, a mediator of notch signaling, is an androgen receptor corepressor. Mol. Cell. Biol. 25, 1425-1436.

Belleudi, F., Purpura, V. and Torrisi, M. R. (2011). The receptor tyrosine kinase FGFR2b/KGFR controls early differentiation of human keratinocytes. PLoS ONE 6, e24194.

Berger, P. L., Frank, S. B., Schulz, V. V., Nollet, E. A., Edick, M. J., Holly, B., Chang, T.-T. A., Hostetter, G., Kim, S. and Miranti, C. K. (2014). Transient induction of ING4 by Myc drives prostate epithelial cell differentiation and its disruption drives prostate tumorigenesis. Cancer Res. 74, 3357-3368.

Berger, P. L., Winn, M. E. and Miranti, C. K. (2017). Miz1, a novel target of ING4, can drive prostate luminal epithelial cell differentiation. Prostate 77, 49-59.

Bertrand, F. E., McCubrey, J. A., Angus, C. W., Nutter, J. M. and Sigounas, G. (2014). NOTCH and PTEN in prostate cancer. Adv. Biol. Regul. 56, 51-65.

Bhat, V., Sun, Y. J., Weger, S. and Raouf, A. (2016). Notch-induced expression of FZD7 requires noncanonical NOTCH3 signaling in human breast epithelial cells. Stem Cells Dev. 25, 522-529.

Brown, D., Hikim, A. P. S., Kovacheva, E. L. and Sinha-Hikim, I. (2009). Mouse model of testosterone-induced muscle fiber hypertrophy: involvement of p38 mitogen-activated protein kinase-mediated Notch signaling. J. Endocrinol. 201, 129-139.

Campeau, E., Ruhl, V. E., Rodier, F., Smith, C. L., Rahmberg, B. L., Fuss, J. O., Campisi, J., Yaswen, P., Cooper, P. K. and Kaufman, P. D. (2009). A versatile viral system for expression and depletion of proteins in mammalian cells. PLoS ONE 4, e6529.

Carvalho, F. L. F., Simons, B. W., Eberhart, C. G. and Berman, D. M. (2014). Notch signaling in prostate cancer: a moving target. Prostate 74, 933-945.

Chen, X., Xu, H., Yuan, P., Fang, F., Huss, M., Vega, V. B., Wong, E., Orlov, Y. L., Zhang, W., Jiang, J. et al. (2008). Integration of external signaling pathways with the core transcriptional network in embryonic stem cells. Cell 133, 1106-1117.

Cisneros, E., Latasa, M. J., García-Flores, M. and Frade, J. M. (2008). Instability of Notch1 and Delta1 mRNAs and reduced Notch activity in vertebrate neuroepithelial cells undergoing S-phase. Mol. Cell. Neurosci. 37, 820-831.

Conacci-Sorrell, M., McFerrin, L. and Eisenman, R. N. (2014). An overview of MYC and its interactome. Cold Spring Harb. Perspect. Med. 4, a014357.

Cress, A. E., Rabinovitz, I., Zhu, W. and Nagle, R. B. (1995). The alpha 6 beta 1 and alpha 6 beta 4 integrins in human prostate cancer progression. Cancer Metastasis Rev. 14, 219-228.

Cuadrado, A. and Nebreda, A. R. (2010). Mechanisms and functions of p38 MAPK signalling. Biochem. J. 429, 403-417.

Cui, H., Kong, Y., Xu, M. and Zhang, H. (2013). Notch3 functions as a tumor suppressor by controlling cellular senescence. Cancer Res. 73, 3451-3459.

Dalrymple, S., Antony, L., Xu, Y., Uzgare, A. R., Arnold, J. T., Savaugeot, J., Sokoll, L. J., De Marzo, A. M. and Isaacs, J. T. (2005). Role of notch-1 and Ecadherin in the differential response to calcium in culturing normal versus malignant prostate cells. Cancer Res. 65, 9269-9279.

Defoiche, J., Zhang, Y., Lagneaux, L., Pettengell, R., Hegedus, A., Willems, L. and Macallan, D. C. (2009). Measurement of ribosomal RNA turnover in vivo by use of deuterium-labeled glucose. Clin. Chem. 55, 1824-1833.

Delmore, J. E., Issa, G. C., Lemieux, M. E., Rahl, P. B., Shi, J., Jacobs, H. M., Kastritis, E., Gilpatrick, T., Paranal, R. M., Qi, J. et al. (2011). BET bromodomain inhibition as a therapeutic strategy to target c-Myc. Cell 146, 904-917 
Deng, G., Ma, L., Meng, Q., Ju, X., Jiang, K., Jiang, P. and Yu, Z. (2015). Notch signaling in the prostate: critical roles during development and in the hallmarks of prostate cancer biology. J. Cancer Res. Clin. Oncol. 142, 531-47.

Edick, M. J., Tesfay, L., Lamb, L. E., Knudsen, B. S. and Miranti, C. K. (2007) Inhibition of integrin-mediated crosstalk with epidermal growth factor receptor/Erk or Src signaling pathways in autophagic prostate epithelial cells induces caspaseindependent death. Mol. Biol. Cell 18, 2481-2490.

Fischer, A., Klattig, J., Kneitz, B., Diez, H., Maier, M., Holtmann, B., Englert, C. and Gessler, M. (2005). Hey basic helix-loop-helix transcription factors are repressors of GATA4 and GATA6 and restrict expression of the GATA target gene ANF in fetal hearts. Mol. Cell. Biol. 25, 8960-8970.

Frank, S. B. and Miranti, C. K. (2013). Disruption of prostate epithelia differentiation pathways and prostate cancer development. Front. Oncol. 3, 273.

Frank, S. B., Schulz, V. V. and Miranti, C. K. (2017). A streamlined method for the design and cloning of shRNAs into an optimized Dox-inducible lentiviral vector BMC Biotechnol. 17, 24.

Furukawa, S., Kawasaki, Y., Miyamoto, M., Hiyoshi, M., Kitayama, J. and Akiyama, T. (2013). The miR-1-NOTCH3-Asef pathway is important for colorecta tumor cell migration. PLOS ONE 8, e80609.

Gagan, J., Dey, B. K., Layer, R., Yan, Z. and Dutta, A. (2012). Notch3 and Mef2c proteins are mutually antagonistic via Mkp1 protein and miR-1/206 microRNAs in differentiating myoblasts. J. Biol. Chem. 287, 40360-40370.

Gebhardt, A., Frye, M., Herold, S., Benitah, S. A., Braun, K., Samans, B., Watt, F. M., Elsässer, H. P. and Eilers, M. (2006). Myc regulates keratinocyte adhesion and differentiation via complex formation with Miz1. J. Cell Biol. 172, 139-149.

Gomi, K., Arbelaez, V., Crystal, R. G. and Walters, M. S. (2015). Activation of NOTCH1 or NOTCH3 signaling skews human airway basal cell differentiation toward a secretory pathway. PLOS ONE 10, e0116507.

Gonsalves, F. C. and Weisblat, D. A. (2007). MAPK regulation of maternal and zygotic Notch transcript stability in early development. Proc. Natl. Acad. Sci. USA 104, 531-536.

Gruber, A. R., Fallmann, J., Kratochvill, F., Kovarik, P. and Hofacker, I. L. (2011) AREsite: a database for the comprehensive investigation of AU-rich elements. Nucleic Acids Res. 39, D66-D69.

Guo, J., Parise, R. A., Joseph, E., Egorin, M. J., Lazo, J. S., Prochownik, E. V. and Eiseman, J. L. (2009). Efficacy, pharmacokinetics, tisssue distribution, and metabolism of the Myc-Max disruptor, 10058-F4 [Z,E]-5-[4-ethylbenzylidine]2-thioxothiazolidin-4-one, in mice. Cancer Chemother. Pharmacol. 63, 615-625.

Harrold, S., Genovese, C., Kobrin, B., Morrison, S. L. and Milcarek, C. (1991). A comparison of apparent mRNA half-life using kinetic labeling techniques vs decay following administration of transcriptional inhibitors. Anal. Biochem. 198, 19-29.

Heer, R., Collins, A. T., Robson, C. N., Shenton, B. K. and Leung, H. Y. (2006) KGF suppresses alpha2beta1 integrin function and promotes differentiation of the transient amplifying population in human prostatic epithelium. J. Cell Sci. 119 1416-1424.

Herrick, D. J. and Ross, J. (1994). The half-life of c-myc mRNA in growing and serum-stimulated cells: influence of the coding and $3^{\prime}$ untranslated regions and role of ribosome translocation. Mol. Cell. Biol. 14, 2119-2128.

Hodkinson, P. S., Elliott, P. A., Lad, Y., McHugh, B. J., MacKinnon, A. C., Haslett, C. and Sethi, T. (2007). Mammalian NOTCH-1 activates beta1 integrins via the small GTPase R-Ras. J. Biol. Chem. 282, 28991-29001.

Huang, M.-J., Cheng, Y.-C., Liu, C.-R., Lin, S. and Liu, H. E. (2006). A smallmolecule c-Myc inhibitor, 10058-F4, induces cell-cycle arrest, apoptosis, and myeloid differentiation of human acute myeloid leukemia. Exp. Hematol. 34, 1480-1489.

Kent, W. J., Sugnet, C. W., Furey, T. S., Roskin, K. M., Pringle, T. H., Zahler, A. M. and Haussler, D. (2002). The human genome browser at UCSC. Genome Res. 12, 996-1006.

Kiec-Wilk, B., Grzybowska-Galuszka, J., Polus, A., Pryjma, J., Knapp, A. and Kristiansen, K. (2010). The MAPK-dependent regulation of the Jagged/Notch gene expression by VEGF, bFGF or PPAR gamma mediated angiogenesis in HUVEC. J. Physiol. Pharmacol. 61, 217-225.

Kim, T.-K., Hemberg, M., Gray, J. M., Costa, A. M., Bear, D. M., Wu, J., Harmin D. A., Laptewicz, M., Barbara-Haley, K., Kuersten, S. et al. (2010). Widespread transcription at neuronal activity-regulated enhancers. Nature 465, 182-187.

Koh, C. M., Bieberich, C. J., Dang, C. V., Nelson, W. G., Yegnasubramanian, S. and De Marzo, A. M. (2010). MYC and prostate cancer. Genes Cancer 1, 617-628.

Kopan, R. and Ilagan, M. X. G. (2009). The canonical Notch signaling pathway: unfolding the activation mechanism. Cell 137, 216-233.

Kwon, O.-J., Valdez, J. M., Zhang, L., Zhang, B., Wei, X., Su, Q., Ittmann, M. M., Creighton, C. J. and Xin, L. (2014). Increased Notch signalling inhibits anoikis and stimulates proliferation of prostate luminal epithelial cells. Nat. Commun 5, 4416.

Kwon, O.-J., Zhang, L. and Xin, L. (2016). Stem cell antigen-1 identifies a distinct androgen-independent murine prostatic luminal cell lineage with bipotent potential. Stem Cells 34, 191-202.

Lam, M. T. Y., Li, W., Rosenfeld, M. G. and Glass, C. K. (2014). Enhancer RNAs and regulated transcriptional programs. Trends Biochem. Sci. 39, 170-182.
Lamb, L. E., Knudsen, B. S. and Miranti, C. K. (2010). E-cadherin-mediated survival of androgen-receptor-expressing secretory prostate epithelial cells derived from a stratified in vitro differentiation model. J. Cell Sci. 123, 266-276.

Litvinov, I. V., Vander Griend, D. J., Xu, Y., Antony, L., Dalrymple, S. L. and Isaacs, J. T. (2006). Low-calcium serum-free defined medium selects for growth of normal prostatic epithelial stem cells. Cancer Res. 66, 8598-8607.

Liu, X. D., Zhang, L. Y., Zhu, T. C., Zhang, R. F., Wang, S. L. and Bao, Y. (2015) Overexpression of miR-34c inhibits high glucose-induced apoptosis in podocytes by targeting Notch signaling pathways. Int. J. Clin. Exp. Pathol. 8, 4525-4534.

üscher, B. and Vervoorts, J. (2012). Regulation of gene transcription by the oncoprotein MYC. Gene 494, 145-160

Magnuson, B., Veloso, A., Kirkconnell, K. S., de Andrade Lima, L. C., Paulsen, M. T., Ljungman, E. A., Bedi, K., Prasad, J., Wilson, T. E. and Ljungman, M. (2015). Identifying transcription start sites and active enhancer elements using BruUV-seq. Sci. Rep. 5, 17978.

Marderosian, M., Sharma, A., Funk, A. P., Vartanian, R., Masri, J., Jo, O. D. and Gera, J. F. (2006). Tristetraprolin regulates Cyclin D1 and c-Myc mRNA stability in response to rapamycin in an Akt-dependent manner via p38 MAPK signaling. Oncogene 25, 6277-6290.

Mathelier, A., Fornes, O., Arenillas, D. J., Chen, C.-Y., Denay, G., Lee, J., Shi, W. Shyr, C., Tan, G., Worsley-Hunt, R. et al. (2016). JASPAR 2016: a major expansion and update of the open-access database of transcription factor binding profiles. Nucleic Acids Res. 44, D110-D115.

Mazzone, M., Selfors, L. M., Albeck, J., Overholtzer, M., Sale, S., Carroll, D. L., Pandya, D., Lu, Y., Mills, G. B., Aster, J. C. et al. (2010). Dose-dependent induction of distinct phenotypic responses to Notch pathway activation in mammary epithelial cells. Proc. Natl. Acad. Sci. USA 107, 5012-5017.

McKeown, M. R. and Bradner, J. E. (2014). Therapeutic strategies to inhibit MYC Cold Spring Harb. Perspect. Med. 4, a014266.

Mori, M., Mahoney, J. E., Stupnikov, M. R., Paez-Cortez, J. R., Szymaniak, A. D. Varelas, X., Herrick, D. B., Schwob, J., Zhang, H. and Cardoso, W. V. (2015) Notch3-Jagged signaling controls the pool of undifferentiated airway progenitors Development 142, 258-267.

Nguyen, B.-C., Lefort, K., Mandinova, A., Antonini, D., Devgan, V., Della Gatta G., Koster, M. I., Zhang, Z., Wang, J., Tommasi di Vignano, A. et al. (2006) Cross-regulation between Notch and p63 in keratinocyte commitment to differentiation. Genes Dev. 20, 1028-1042

Ohashi, S., Natsuizaka, M., Yashiro-Ohtani, Y., Kalman, R. A., Nakagawa, M. Wu, L., Klein-Szanto, A. J., Herlyn, M., Diehl, J. A., Katz, J. P. et al. (2010) $\mathrm{NOTCH} 1$ and NOTCH3 coordinate esophageal squamous differentiation through a CSL-dependent transcriptional network. Gastroenterology 139 2113-2123.

Ong, C.-T., Cheng, H.-T., Chang, L.-W., Ohtsuka, T., Kageyama, R., Stormo, G. D. and Kopan, R. (2006). Target selectivity of vertebrate notch proteins. Collaboration between discrete domains and CSL-binding site architecture determines activation probability. J. Biol. Chem. 281, 5106-5119.

Ortica, S., Tarantino, N., Aulner, N., Israel, A. and Gupta-Rossi, N. (2014). The 4 Notch receptors play distinct and antagonistic roles in the proliferation and hepatocytic differentiation of liver progenitors. FASEB J. 28, 603-614.

Ousset, M., Van Keymeulen, A., Bouvencourt, G., Sharma, N., Achouri, Y., Simons, B. D. and Blanpain, C. (2012). Multipotent and unipotent progenitors contribute to prostate postnatal development. Nat. Cell Biol. 14, 1131-1138.

Park, J.-S., Kim, Y.-S. and Yoo, M.-A. (2009). The role of p38b MAPK in age-related modulation of intestinal stem cell proliferation and differentiation in Drosophila. Aging (Albany NY) 1, 637-651.

Paulsen, M. T., Veloso, A., Prasad, J., Bedi, K., Ljungman, E. A., Magnuson, B., Wilson, T. E. and Ljungman, M. (2014). Use of Bru-Seq and BruChase-Seq fo genome-wide assessment of the synthesis and stability of RNA. Methods 67 $45-54$

Pedrosa, A.-R., Graça, J. L., Carvalho, S., Peleteiro, M. C., Duarte, A. and Trindade, A. (2016). Notch signaling dynamics in the adult healthy prostate and in prostatic tumor development. Prostate 76, 80-96.

Rangarajan, A., Talora, C., Okuyama, R., Nicolas, M., Mammucari, C., Oh, H. Aster, J. C., Krishna, S., Metzger, D., Chambon, P. et al. (2001). Notch signaling is a direct determinant of keratinocyte growth arrest and entry into differentiation. EMBO J. 20, 3427-3436.

Romano, R.-A., Smalley, K., Magraw, C., Serna, V. A., Kurita, T., Raghavan, S and Sinha, S. (2012). DeltaNp63 knockout mice reveal its indispensable role as a master regulator of epithelial development and differentiation. Development 139 $772-782$

Shou, J., Ross, S., Koeppen, H., de Sauvage, F. J. and Gao, W. Q. (2001) Dynamics of notch expression during murine prostate development and tumorigenesis. Cancer Res. 61, 7291-7297.

The ENCODE Project Consortium (2012). An integrated encyclopedia of DNA elements in the human genome. Nature 489, 57-74.

Tokar, E. J., Ancrile, B. B., Cunha, G. R. and Webber, M. M. (2005). Stem progenitor and intermediate cell types and the origin of human prostate cancer. Differentiation 73, 463-473. 
Uzgare, A. R., Xu, Y. and Isaacs, J. T. (2004). In vitro culturing and characteristics of transit amplifying epithelial cells from human prostate tissue. J. Cell. Biochem. 91 196-205.

Valdez, J. M., Zhang, L., Su, Q., Dakhova, O., Zhang, Y., Shahi, P., Spencer, D. M., Creighton, C. J., Ittmann, M. M. and Xin, L. (2012). Notch and TGFbeta form a reciprocal positive regulatory loop that suppresses murine prostate basal stem/progenitor cell activity. Cell Stem Cell 11, 676-688.

Vander Griend, D. J., Litvinov, I. V. and Isaacs, J. T. (2014). Conversion of androgen receptor signaling from a growth suppressor in normal prostate epithelial cells to an oncogene in prostate cancer cells involves a gain of function in c-Myc regulation. Int. J. Biol. Sci. 10, 627-642.

Wang, J., Ma, X., Jones, H. M., Chan, L. L.-Y., Song, F., Zhang, W., Bae-Jump, V. L. and Zhou, C. (2014). Evaluation of the antitumor effects of c-Myc-Max heterodimerization inhibitor 100258-F4 in ovarian cancer cells. J. Transl. Med. 12, 226.
Wang, X., Shen, Q. W., Wang, J., Zhang, Z., Feng, F., Chen, T., Zhang, Y., Wei, H., Li, Z., Wang, X. et al. (2016). KLF7 regulates satellite cell quiescence in response to extracellular signaling. Stem Cells 34, 1310-1320.

Weng, A. P., Millholland, J. M., Yashiro-Ohtani, Y., Arcangeli, M. L., Lau, A., Wai, C., Del Bianco, C., Rodriguez, C. G., Sai, H., Tobias, J. et al. (2006). c-Myc is an important direct target of Notch1 in T-cell acute lymphoblastic leukemia/ lymphoma. Genes Dev. 20, 2096-2109.

Xiao, L., Feng, Q., Zhang, Z., Wang, F., Lydon, J. P., Ittmann, M. M., Xin, L., Mitsiades, N. and He, B. (2016). The essential role of GATA transcription factors in adult murine prostate. Oncotarget 7, 47891-47903.

Yoshida, Y., Hayashi, Y., Suda, M., Tateno, K., Okada, S., Moriya, J., Yokoyama, M., Nojima, A., Yamashita, M., Kobayashi, Y. et al. (2014). Notch signaling regulates the lifespan of vascular endothelial cells via a p16-dependent pathway. PLOS ONE 9, e100359. 